\title{
STABILITY ANALYSIS AND SEMI-ANALYTIC SOLUTION TO A SEIR-SEI MALARIA TRANSMISSION MODEL USING HE'S VARIATIONAL ITERATION METHOD
}

\author{
1. AKINFE, Timilehin Kingsley* \\ 2. LOYINMI, Adedapo Chris \\ ${ }^{1,2}$ Department of Mathematics, Tai Solarin University of Education, Ijagun, \\ Ijebu ode, Ogun state, Nigeria; akinfetimilehin@gmail.com*; loyinmiac@tasued.edu.ng \\ ${ }^{1}$ ORC-ID: 0000-0002-5308-7053*; ${ }^{2}$ ORC-ID: 0000-0002-6171-4256 \\ *Correspondence: akinfetimilehin@gmail.com
}

\begin{abstract}
:
We have considered a SEIR-SEI Vector-host mathematical model which captures malaria transmission dynamics, described and built on 7-dimensional nonlinear ordinary differential equations. We compute the basic reproduction number of the model; examine the positivity and boundedness of the model compartments in a region using well established methods viz: Cauchy's differential theorem, Birkhoff \& Rota's theorem which verifies and reveals the wellposedness, and carrying capacity of the model respectively, the existence of the Disease-Free (DFE) and Endemic (EDE) equilibrium points were determined and examined

Using the Gaussian elimination method and the Routh-hurwitz criterion, we convey stability analyses at DFE and EDE points which indicates that the DFE (malaria-free) and the EDE (epidemic outbreak) point occurs when the basic reproduction number is less than unity (one) and greater than unity (one) respectively.

We obtain a solution to the model using the Variational iteration method (VIM) (an unprecedented method) to each population compartments and verify the efficacy, reliability and validity of the proposed method by comparing the respective solutions via tables and combined plots with the computer in-built Runge-kutta-Felhberg of fourth-fifths order (RKF-45).
\end{abstract}


We illustrate the combined plot profiles of each compartment in the model, showing the dynamic behavior of these compartments; then we speculate that VIM is efficient and capable to conduct analysis on Malaria models and other epidemiological models.

\section{Keywords:}

SEIR-SEI, Basic Reproduction number, Disease-Free equilibrium point (DFE), Endemic equilibrium point, Stability, Variational iteration method (VIM), Runge-Kutta-Felhberg (RKF45).

\section{Introduction}

Malaria is a mosquito-borne infectious disease that is life threatening to humans and other animals (Malaria fact sheet, 2014) [16]. This infectious disease is widely spread throughout the globe and predominantly present in tropical and sub-tropical regions of the earth including some parts of Europe.

The wide spread of this vector-borne disease (malaria) has urged numerous researchers and health organizations to study the epidemiology and transmission dynamics of the disease; so as to be able to implement an appropriate intervention strategy on its ubiquitous nature.

Because of its nature of being a fatal disease, this is why $25^{\text {th }}$ of April is set aside as the world's annual malaria day for the global alertness against the disease. Malaria causes symptoms that typically include fever, tiredness, vomiting, and headaches (Caraballo, 2014). In severe cases it can cause yellow skin, seizures, coma, or death. (Caraballo, 2014) [15]. These symptoms usually begin ten to fifteen days after being bitten by an infected mosquito and if not properly treated, people may have recurrences of the disease months later (Malaria fact sheet W.H.O, 2014) [16]. Malaria is caused by single-celled microorganisms of the plasmodium group (Malaria Fact sheet W.H.O, 2014). The disease is most commonly spread by an infected female Anopheles mosquito. The mosquito bite introduces the parasites from the mosquito's saliva into the Host (Human). There are five different plasmodium species leading to malaria infection and disease among humans; These are: Plasmodium Falciparum (P. falciparum), Plasmodium vivax (P. vivax), Plasmodium Ovale (P. ovale), Plasmodium malariae (P. malariae), Plasmodium knowlesi (P. knowlesi) [8]. 
Most deaths are caused by P. falciparum as it is the most dangerous of all plamodium species [8, 13]. P. vivax, P. ovale, and P. malariae generally cause milder form of malaria while the P. knowlesi rarely cause disease in humans. This P. falciparum is mainly found in Africa as it is common and causing deaths worldwide. In addition, Plasmodium knowlesi is a type of malaria that infects macaques in Southeast Asia; also infect humans causing malaria that is transmitted from animal to human (zoonotic malaria) $[8,13-14]$.

WHO Malaria report (2013) shows that approximately $80 \%$ of malaria cases and $90 \%$ of deaths are estimated to occur in most countries of this sub-Saharan Africa [9]. In 2015, WHO estimates that 212 million clinical cases of malaria occurred and 429,000 people died of malaria, most of them were children in Africa [10]. The world Malaria Report in 2018 [38] shows an unprecedented period of success in global malaria control. An estimated 219 million cases of malaria occurred worldwide (95\% confidence interval (CI): 203-262 million), compared with 239 million cases in 2010 (95\% CI: 219-285 million) [11] and 217 million cases in 2016 (95\% CI: 200-259 million) [12] with 92\% cases in the African region, 5\% in the South-East Asia region and $2 \%$ in the WHO Eastern Mediterranean region.

Very recently, in the common wealth malaria reports (April, 2019) [39]; a historic partnership of governments, civil society, the private sector and multilateral organizations, came together in London for a momentous malaria summit. Delivering US \$4.1 billion for the global malaria fight and two days later at the commonwealth heads of Government meeting (CHOGM), all 53 leaders committed to halve malaria in the commonwealth within five years.

The report here shows that the commonwealth countries: The Gambia, Belize, Bangladesh, India, Malaysia, Mozambique and Nigeria are already on a trajectory to achieve the target to halve malaria in 2023. See [39].

Due to the everyday attempt to control the epidemic and prevalent nature of malaria, several models have been developed by mathematicians; so as to understand the transmission dynamics of this infectious disease and implement a control strategy. Majority of these models are being described by differential equations of the nonlinear type. The first malaria model for malaria transmission and control was by Ronald Ross [4] which was later improved by Macdonald 
(1957) [5] considering some biological assumptions. Since then, many models have been developed like Ngwa and Shu (1999) [18] Jia Li (2011) [3], Prashant Goswami et al (2012) [20], Olaniyi S and Obabiyi (2013) [2], Shah NH and Gupta. J (2013) [21], Hal-Feng Huo and Guangming Qiu(2014) [23], Altaf Khan et al (2015) [22], Oti eno (2016), Osman et al (2017) [1], Osman et al (2018) [24], Traore Bakare (2018) [7] to mention a few. Researchers and mathematicians have endeavored to proffer solution to these models including that of malaria via different methods so as to understand the transmission dynamics better in Nigar Ali et al. (2019) using Adomian Decomposition method [25], Abioye adesoye idowu et al (2018) using Differential transform method [28], Peter olumuyiwa james et al (2018) solved using Multi-step Homotopy analysis method [30]. Morufu oyedunsi olayiwola (2017) using the Variational iteration method solved a SEIRS epidemic model [26]. Yullita molliq Rangkuti (2014) obtained a numerical analytical solution of SIR model of Dengue fever disease in South sulawesi using HPM and VIM [19], Fazal Haq et al (2017) by Laplace Adomian decomposition method solved an epidemic model of a vector borne disease [17].

Of all the semi-analytical methods implemented to solve epidemic models including malaria, none have solved the malaria model using the variational iteration method and as a result, less attention has been paid using this method on malaria models. This method is unprecedented.

The main reason of this paper is to validate the efficiency of variational iteration method and also speculate its capability as alternative approach in solving and analyzing epidemiological models including malaria.

The huge advantage of this method over other methods include: the simplicity and straightforwardness, less computational stress or efforts of the method with no linearization of the nonlinear term, no computation of Adomian or He's polynomials, yet yielding highly accurate and rapidly convergent results devoid of errors when compared numerically and graphically. In this research, we consider an existing SEIR model of Osman et al (2017), conduct a stability analysis, and then obtain semi-analytic solution via Variational iteration method (VIM).

The model presented here in this research is of two compartmental system of nonlinear ordinary differential equation involving the host which is the human and the Vector which is the mosquito. The human (host) is described by four differential equations and the mosquito by three differential equations. 
The subsequent organization of this research work is structured as follows: Section 2 elucidates the compartmental model of the malaria transmission dynamics as well as the flow diagram of the model; Section 3 focuses on the mathematical analysis of the model which includes the analysis on the feasible region $\Gamma$ of the model, so as to verify the epidemiological validity of the model; the disease-free equilibrium point (DFE), basic reproduction number, the endemic equilibrium point (EDE), stability of the DFE via Gaussian elimination method and the EDE with theorems, lemmas, and proofs were all computed here.

Semi-analytic solution was then proffered to the seven (7) compartments of the vector-host model using He's variational iteration method (VIM) in Section 4.

Lastly, numerical result comparison were made for the solved compartments via tables and combined plots of Runge-Kutta-Felhberg 45 (RKF-45) and VIM, results were then interpreted and discussed before the final conclusion in section 5 and 6 respectively.

\section{The Model}

The model consists of two classes of population, the human population and the mosquito population. The human $N$ population is subdivided into four compartments, the susceptible, the exposed, the infected, and the recovered. While the mosquito $N$ population is subdivided into three compartments, the susceptible, the exposed, the infected as it is assumed that mosquitoes don't recover. We then have that the SEIR model for the humans (host) and the SEI model for the mosquito (vector). (Table 1)

\subsection{Model Assumptions}

The Population of the susceptible human $S_{H}(t)$ is increased by the recruitment of individuals at a rate $\Lambda_{H}$, and by the recovered individuals returning back to the compartment due to loss of immunity at a rate $\rho$, they acquire infection at a rate $\beta_{H}$, the population is then decreased by natural death of humans at a rate $\mu_{H}$. (Fig 1) The population of the Exposed human $E_{H}(t)$ is generated by the infection of the susceptible individuals at a rate $\beta_{H}$, decreased by 
humans whose infection has developed to the infectious compartment at a rate $\alpha_{1}$, and further decreased by natural death $\mu_{H}$. (Fig 2)

The population of the infected $I_{H}(t)$ is generated by humans who are infectious at a rate $\alpha_{1}$, increased by newborn baby with infection at rate $\psi$, then decreased by natural death $\mu_{H}$, malaria induced death, and humans who have recovered at rates $\mu_{H}, \delta$, and $\alpha_{2}$ respectively. (Fig 3)

The Recovered population $\mathrm{R}_{\mathrm{H}}(t)$ is generated by those who are infected but are being treated and recovering from malaria at a rate $\alpha_{2}$. It is then decreased by those who die naturally and lose their immunity at rates $\mu_{H}$ and $\rho$ respectively. (Fig 4)

The susceptible mosquito population $S_{V}(t)$ is generated by the recruitment of mosquitoes into the compartment at a rate $\Lambda_{V}$, decreased by infection and death by natural cause with rates $\beta_{V}$ and $\mu_{V}$. (Fig 5)

The Exposed mosquito's population $E_{V}(t)$ is generated by susceptible mosquitoes exposed to the malaria pathogen infection at a rate $\beta_{V}$, decreased by mosquitoes that have developed into the infectious state, and by natural cause at rates $\alpha_{3}$, and $\mu_{V}$. (Fig 6)

The Infected mosquito's population $I_{V}(t)$ is generated by exposed mosquito whose state has moved to the infectious state at the rate $\alpha_{3}$, and decreased by natural cause $\mu_{V}$. (Fig 7)

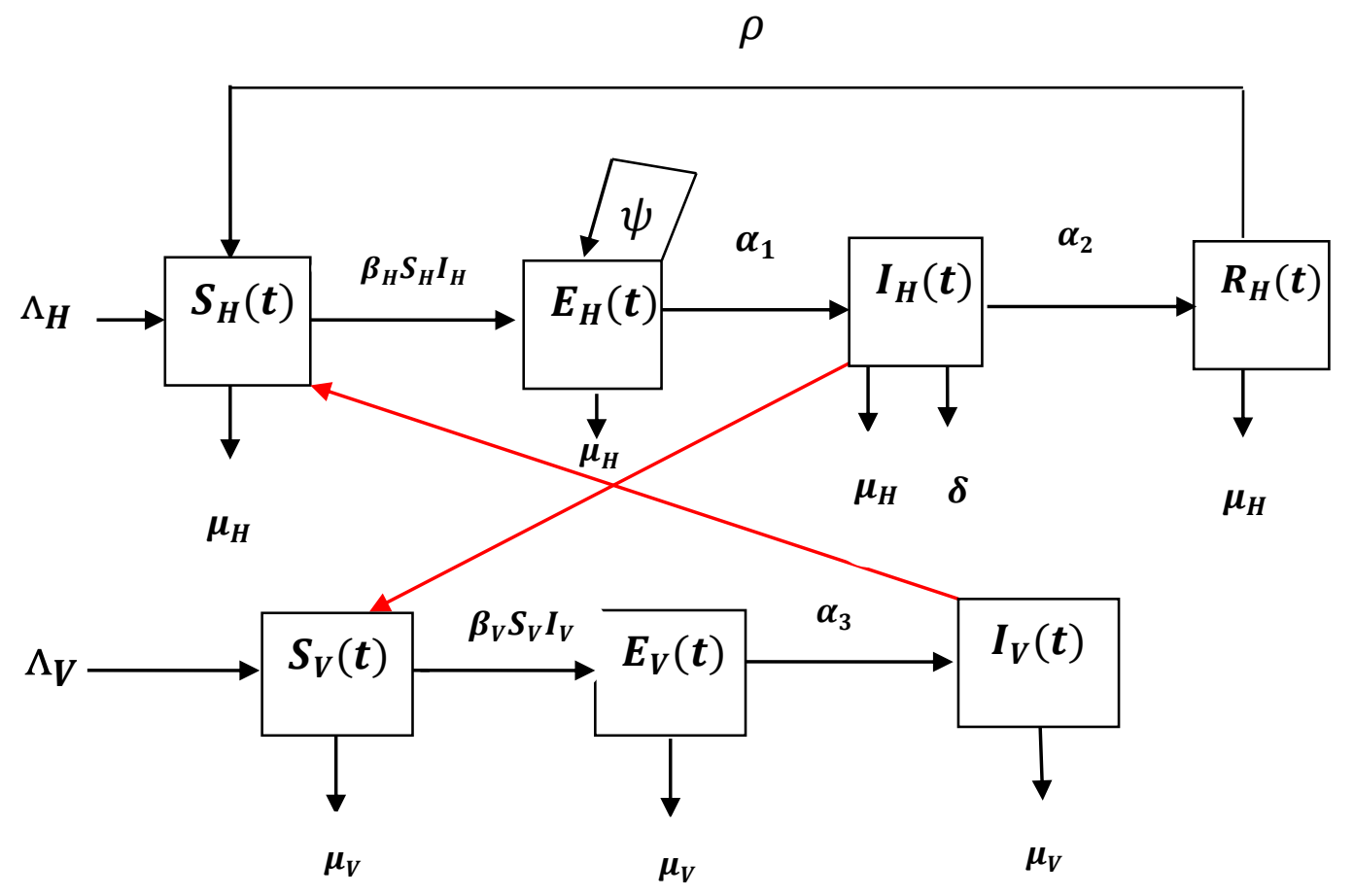




$$
\begin{aligned}
& \frac{d S_{H}(t)}{d t}=\Lambda_{H}-\beta_{H} S_{H} I_{H}-\mu_{H} S_{H}+\rho R_{H} \\
& \frac{d E_{H}(t)}{d t}=\beta_{H} S_{H} I_{H}-\left(\alpha_{1}+\mu_{H}\right) E_{H} \\
& \frac{d I_{H}(t)}{d t}=\alpha_{1} E_{H}-\left(\alpha_{2}+\mu_{H}+\delta\right) I_{H}+\psi I_{H} \\
& \frac{d R_{H}(t)}{d t}=\alpha_{2} I_{H}-\left(\mu_{H}+\rho\right) R_{H} \\
& \frac{d S_{V}(t)}{d t}=\Lambda_{V}-\beta_{V} S_{V} I_{V}-\mu_{V} S_{V} \\
& \frac{d E_{V}(t)}{d t}=\beta_{V} S_{V} I_{V}-\left(\alpha_{3}+\mu_{V}\right) E_{V} \\
& \frac{d I_{V}(t)}{d t}=\alpha_{3} E_{V}-\mu_{V} I_{V}
\end{aligned}
$$

Table 1: State Variables and parameter description of the SEIR-SEI model

\section{State Variables Description}

\begin{tabular}{cl}
\hline $\boldsymbol{S}_{\boldsymbol{H}}(\boldsymbol{t})$ & Susceptible Human at time t \\
$\boldsymbol{E}_{\boldsymbol{H}}(\boldsymbol{t})$ & Exposed Human at time t \\
$\boldsymbol{I}_{\boldsymbol{H}}(\boldsymbol{t})$ & Infected Human at time t \\
$\boldsymbol{R}_{\boldsymbol{H}}(\boldsymbol{t})$ & Recovered Human at time t \\
$\boldsymbol{S}_{\boldsymbol{V}}(\boldsymbol{t})$ & Susceptible mosquito at time t \\
$\boldsymbol{E}_{\boldsymbol{V}}(\boldsymbol{t})$ & Exposed mosquito at time t \\
$\boldsymbol{I}_{\boldsymbol{V}}(\boldsymbol{t})$ & Infected mosquito at time t \\
& \\
Parameters & Description \\
$\Lambda_{\boldsymbol{H}}$ & Recruitment rate of Humans \\
$\Lambda_{V}$ & Recruitment rate of Mosquitoes \\
\hline
\end{tabular}




$\begin{array}{ll}\boldsymbol{\alpha}_{\mathbf{1}} & \text { Development from exposure to being infectious (Humans) } \\ \boldsymbol{\alpha}_{2} & \text { Recovery rate of Humans } \\ \boldsymbol{\alpha}_{3} & \text { Development from exposure to being infectious (mosquito) } \\ \boldsymbol{\mu}_{\boldsymbol{H}} & \text { Natural Death of Humans } \\ \boldsymbol{\mu}_{\boldsymbol{V}} & \text { Malaria induced death rate for Humans } \\ \boldsymbol{\delta} & \text { Probability of transmission from an infectious mosquito to a susceptible } \\ \boldsymbol{q}_{\boldsymbol{H}} & \text { Human } \\ \boldsymbol{q}_{\boldsymbol{V}} & \text { Probability of Transmission from an infectious Human to a susceptible } \\ \boldsymbol{\psi}_{\boldsymbol{H}} & \text { mosquito } \\ \boldsymbol{\beta}_{\boldsymbol{H}} & \text { Mosquito Biting Rate } \\ \boldsymbol{\eta}_{\boldsymbol{V}} & \text { Infection rate of Humans }\left(q_{H} \times \eta_{V}\right) \\ & \text { Loss of Immunity for Humans } \\ & \end{array}$

\subsection{MATHEMATICAL ANALYSIS OF THE MODEL}

\subsection{Positivity and Boundedness of Solution}

Here, results are presented and verifications are made as to guarantee that the malaria model governed by the system (1) is epidemiologically and mathematically well-posed in a feasible region $\Gamma$; given by: $\Gamma=\Gamma_{H} \times \Gamma_{V} \subset \mathfrak{R}_{+}^{4} \times \mathfrak{R}_{+}^{3}$ 
Where,

$$
\begin{aligned}
& \Gamma_{H}=\left\{\left(S_{H}, E_{H}, I_{H}, R_{H}\right) \in \mathfrak{R}_{+}^{4}: S_{H}+E_{H}+I_{H}+R_{H} \leq \frac{\Lambda_{H}}{\mu_{H}}, S_{H}>0, E_{H} \geq 0, I_{H} \geq 0, R_{H} \geq 0\right\}, \\
& \Gamma_{V}=\left\{\left(S_{V}, E_{V}, I_{V}\right) \in \mathfrak{R}_{+}^{3}: S_{V}+E_{V}+I_{V} \leq \frac{\Lambda_{V}}{\mu_{V}}, S_{V}>0, E_{V} \geq 0, I_{V} \geq 0\right\} .
\end{aligned}
$$

\subsubsection{Theorem 1:}

The feasible region of the system (1) given by

$$
\Gamma=\left\{\begin{array}{l}
\left(S_{H}, E_{H}, I_{H}, R_{H}, S_{V}, E_{V}, I_{V}\right): S_{H}(t)+E_{H}(t)+I_{H}(t)+R_{H}(t) \leq \frac{\Lambda_{H}}{\mu_{H}} ; S_{V}(t)+E_{V}(t)+I_{V}(t) \leq \frac{\Lambda_{V}}{\mu_{V}} ; S_{H}>0, \\
E_{H} \geq 0, I_{H} \geq 0, R_{H} \geq 0, S_{V}>0, E_{V} \geq 0, I_{V} \geq 0
\end{array}\right\}
$$

is a positive invariant set and Bounded.

Proof: Let us consider the Host Population governed by the system

$$
\begin{aligned}
& \frac{d S_{H}(t)}{d t}=\Lambda_{H}-\beta_{H} S_{H} I_{H}-\mu_{H} S_{H}+\rho R_{H} \\
& \frac{d E_{H}(t)}{d t}=\beta_{H} S_{H} I_{H}-\left(\alpha_{1}+\mu_{H}\right) E_{H} \\
& \frac{d I_{H}(t)}{d t}=\alpha_{1} E_{H}-\left(\alpha_{2}+\mu_{H}+\delta\right) I_{H}+\psi I_{H} \\
& \frac{d R_{H}(t)}{d t}=\alpha_{2} I_{H}-\left(\mu_{H}+\rho\right) R_{H}
\end{aligned}
$$

$N_{H}=S_{H}(t)+E_{H}(t)+I_{H}(t)+R_{H}(t)$ is the human net population.

Now from the derivatives of sums;

$$
\frac{d N_{H}(t)}{d t}=\frac{d S_{H}(t)}{d t}+\frac{d E_{H}(t)}{d t}+\frac{d I_{H}(t)}{d t}+\frac{d R_{H}(t)}{d t}
$$

This implies that,

$$
\begin{aligned}
& \frac{d N_{H}(t)}{d t}=\left(\Lambda_{H}-\beta_{H} S_{H} I_{H}-\mu_{H} S_{H}+\rho R_{H}\right)+\left(\beta_{H} S_{H} I_{H}-\left(\alpha_{1}+\mu_{H}\right) E_{H}\right) \\
& +\left(\alpha_{1} E_{H}-\left(\alpha_{2}+\mu_{H}+\delta\right) I_{H}+\psi I_{H}\right)+\left(\alpha_{2} I_{H}-\left(\mu_{H}+\rho\right) R_{H}\right)
\end{aligned}
$$

Then,

$$
\begin{aligned}
& \frac{d N_{H}(t)}{d t}=\Lambda_{H}-\mu_{H}\left(S_{H}+E_{H}+I_{H}+R_{H}\right)-(\delta-\psi) I_{H} \\
& \therefore \frac{d N_{H}(t)}{d t}=\Lambda_{H}-\mu_{H} N_{H}-(\delta-\psi) I_{H}
\end{aligned}
$$


This implies that $\frac{d N_{H}(t)}{d t} \leq \Lambda_{H}-\mu_{H} N_{H}$ when we remove the parameter $(\delta-\psi) I_{H}$.

$$
\therefore \frac{d N_{H}(t)}{d t}+\mu_{H} N_{H} \leq \Lambda_{H}
$$

By solving the first order linear differential inequality (4) using integrating factor method we have;

$$
N_{H}(t) \leq \frac{\Lambda_{H}}{\mu_{H}}+p e^{-\mu_{H} t}
$$

Where $p$ is a constant of integration.

Then by applying Birkhoff and Rota's theorem [31] on the differential inequality (5), it follows that

$$
\lim _{t \rightarrow \infty} N_{H}(t) \leq \frac{\Lambda_{H}}{\mu_{H}}
$$

This is commonly known as the carrying capacity of the system and hence shows Boundedness.

It then follows that $N_{H}(t)=\left[\left\{S_{H}(t)+E_{H}(t)+I_{H}(t)+R_{H}(t)\right\} \in \mathfrak{R}_{+}^{4} \leq \frac{\Lambda_{H}}{\mu_{H}}\right]$

This proves the boundedness of the solution inside the region $\Gamma_{H}$

Now for other classes of the population we have;

\subsubsection{Other Compartments}

We consider the rate of change of the population in the Susceptible Human compartment

$$
\begin{aligned}
& \frac{d S_{H}(t)}{d t}=\Lambda_{H}-\beta_{H} S_{H} I_{H}-\mu_{H} S_{H}+\rho R_{H} \\
& \Rightarrow \frac{d S_{H}(t)}{d t}+\left(\mu_{H}+\beta_{H} I_{H}\right) S_{H}=\Lambda_{H}+\rho R_{H}
\end{aligned}
$$

Let $\left(\mu_{H}+\beta_{H} I_{H}\right)=\varphi_{H}$,

Then, $\frac{d S_{H}(t)}{d t}+\phi_{H} S_{H}=\Lambda_{H}+\rho R_{H}$

We then can write that 


$$
\frac{d S_{H}(t)}{d t}+\varphi_{H} S_{H}>0
$$

By separation of variables we obtain that;

$$
\begin{aligned}
& \int \frac{d S_{H}(t)}{S_{H}}>\int-\varphi_{H} d t \\
& \Rightarrow \ln \left(S_{H}(t)\right)>-\phi_{H} t+c \\
& \therefore S_{H}(t)>e^{-\phi_{H} t} \cdot e^{c}
\end{aligned}
$$

Let $A=e^{c}$ then we have; $S_{H}(t)>A e^{-\phi_{H} t}$

At the initial state when $t=0, S_{H}(0)>A>0$.

$\therefore S_{H}(t)=S_{H}(0) e^{-\phi_{H} t}=A e^{-\phi_{H} t}>0$ Holds and this implies that $S_{H}(t)>0$ holds.

Indicating that $S_{H}(t)$ stays and remains positive.

Similarly, we consider the non-linear ODE for the exposed human

$$
\begin{aligned}
& \frac{d E_{H}(t)}{d t}=\beta_{H} S_{H} I_{H}-\left(\alpha_{1}+\mu_{H}\right) E_{H} \\
& \therefore \frac{d E_{H}(t)}{d t}+\left(\alpha_{1}+\mu_{H}\right) E_{H}=\beta_{H} S_{H} I_{H}
\end{aligned}
$$

From $\beta_{H} S_{H} I_{H}$ on the right hand side of the equation (11), we have that $S_{H}>0, S_{H} \neq 0$ from our previous proof. Now for $I_{H} \geq 0$ we have that $\frac{d E_{H}(t)}{d t}+\left(\alpha_{1}+\mu_{H}\right) E_{H}=0$ when $I_{H}=0$ and

$$
\begin{array}{r}
\frac{d E_{H}(t)}{d t}+\left(\alpha_{1}+\mu_{H}\right) E_{H}>0 \text { when } I_{H}>0 \\
\therefore \frac{d E_{H}(t)}{d t}+\left(\alpha_{1}+\mu_{H}\right) E_{H} \geq 0
\end{array}
$$

Solving the differential inequality (12) using separation of variable We have

$$
\int \frac{d E_{H}(t)}{E_{H}} \geq \int-\left(\alpha_{1}+\mu_{H}\right) d t
$$


Let $\xi_{H}=\left(\alpha_{1}+\mu_{H}\right)$

$$
\begin{aligned}
& \Rightarrow \int \frac{d E_{H}(t)}{E_{H}} \geq \int-\xi_{H} d t \\
& \therefore \ln \left(E_{H}\right) \geq-\xi_{H} t+c \\
& \Rightarrow E_{H}(t) \geq e^{-\xi_{H} t} \cdot e^{c}
\end{aligned}
$$

When $t=0$, we have

$$
\begin{aligned}
& E_{H}(0) \geq A e^{0}=A \\
& \therefore E_{H}(0)=A \geq 0 \\
& \Rightarrow E_{H}(t)=E_{H}(0) e^{-\xi_{H} t} \geq 0
\end{aligned}
$$

Hence, $E_{H}(t) \geq 0$ Holds.

Similarly, we consider the nonlinear differential equations of other state variables $I_{H}(t)$ and $R_{H}(t)$ of the Infected and the recovered class; we let $\gamma_{H}=\left(\alpha_{2}+\mu_{H}+\delta\right)$ and $\varepsilon_{H}=\left(\mu_{H}+\rho\right)$ respectively and solve the differential inequalities $\frac{d I_{H}}{d t}+\gamma_{H} I_{H} \geq 0, \frac{d R_{H}}{d t}+\varepsilon_{H} R_{H} \geq 0$ with the initial conditions.

We obtain the solutions to the ODEs and we have that $I_{H}(t) \geq 0$ and $R_{H}(t) \geq 0$ hold respectively.

\subsubsection{Mosquito Model (Vector)}

We consider the governing equation of the vector (SEI) model which is the Mosquito.

$$
\begin{aligned}
& \frac{d S_{V}(t)}{d t}=\Lambda_{V}-\beta_{V} S_{V} I_{V}-\mu_{V} S_{V} \\
& \frac{d E_{V}(t)}{d t}=\beta_{V} S_{V} I_{V}-\left(\alpha_{3}+\mu_{V}\right) E_{V} \\
& \frac{d I_{V}(t)}{d t}=\alpha_{3} E_{V}-\mu_{V} I_{V}
\end{aligned}
$$

The total Population density gives

$$
N_{V}(t)=S_{V}(t)+E_{V}(t)+I_{V}(t)
$$

\section{From Cauchy's differential theorem,}




$$
\frac{d N_{V}(t)}{d t}=\frac{\partial N_{V}(t)}{\partial S_{V}} \cdot \frac{d S_{V}}{d t}+\frac{\partial N_{V}(t)}{\partial E_{V}} \cdot \frac{d E_{V}}{d t}+\frac{\partial N_{V}(t)}{\partial I_{V}} \cdot \frac{d I_{V}}{d t}
$$

We have that

$$
\begin{gathered}
\frac{\partial N_{V}(t)}{\partial S_{V}}=\frac{\partial N_{V}(t)}{\partial E_{V}}=\frac{\partial N_{V}(t)}{\partial I_{V}}=1, \\
\therefore \frac{d N_{V}(t)}{d t}=\frac{d S_{V}}{d t}+\frac{d E_{V}}{d t}+\frac{d I_{V}}{d t} \\
\therefore \frac{d N_{V}(t)}{d t}=\left(\Lambda_{V}-\beta_{V} S_{V} I_{V}-\mu_{V} S_{V}\right)+\left(\beta_{V} S_{V} I_{V}-\left(\alpha_{3}+\mu_{V}\right) E_{V}\right)+\left(\alpha_{3} E_{V}-\mu_{V} I_{V}\right)
\end{gathered}
$$

We then have,

$$
\therefore \frac{d N_{V}(t)}{d t}+\mu_{V} N_{V} \leq \Lambda_{V}
$$

By solving the differential inequality by method of integrating factor and apply Birkhoff and Rota's theorem [31]

$$
\lim _{t \rightarrow \infty} N_{V}(t) \leq \frac{\Lambda_{V}}{\mu_{V}}
$$

It then follows that $N_{V}(t)=S_{V}(t)+E_{V}(t)+I_{V}(t) \leq \frac{\Lambda_{V}}{\mu_{V}}$

This proves boundedness.

Similarly as the Host model, $S_{V}(t)>0, E_{V}(t) \geq 0, I_{V}(t) \geq 0$ holds for the mosquito population.

This completely proves our theorem 1.

\subsection{Disease-Free equilibrium points and the Reproduction Number}

The points at which the differential equation is equal to zero are referred to as the equilibrium points or steady-state solutions.

The model consists of just two equilibrium points which is the disease-free and the Endemic equilibrium points

The point or time at which the disease wiped out and the entire population is susceptible is the

Disease-free equilibrium point while the point at which the disease persists in the population (Epidemic outbreak) is the Endemic equilibrium point. 
At Equilibrium,

$$
\frac{d S_{H}(t)}{d t}=\frac{d E_{H}(t)}{d t}=\frac{d I_{H}(t)}{d t}=\frac{d R_{H}(t)}{d t}=\frac{d S_{V}(t)}{d t}=\frac{d E_{V}(t)}{d t}=\frac{d I_{V}(t)}{d t}=0
$$

By substituting (21) into the system of equations (1),

$$
\begin{aligned}
& 0=\Lambda_{H}-\beta_{H} S_{H}^{0} I_{H}^{0}-\mu_{H} S_{H}^{0}+\rho R_{H}^{0} \\
& 0=\beta_{H} S_{H}^{0} I_{H}^{0}-\left(\alpha_{1}+\mu_{H}\right) E_{H}^{0} \\
& 0=\alpha_{1} E_{H}^{0}-\left(\alpha_{2}+\mu_{H}+\delta\right) I_{H}^{0}+\psi I_{H}^{0} \\
& 0=\alpha_{2} I_{H}^{0}-\left(\mu_{H}+\rho\right) R_{H}^{0} \\
& 0=\Lambda_{V}-\beta_{V} S_{V}^{0} I_{V}^{0}-\mu_{V} S_{V}^{0} \\
& 0=\beta_{V} S_{V}^{0} I_{V}^{0}-\left(\alpha_{3}+\mu_{V}\right) E_{V}^{0} \\
& 0=\alpha_{3} E_{V}^{0}-\mu_{V} I_{V}^{0}
\end{aligned}
$$

Then the DFE for the SEIR-SEI system is given by:

$$
E^{0}=\left(S_{H}^{0}, E_{H}^{0}, I_{H}^{0}, R_{H}^{0}, S_{V}^{0}, E_{V}^{0}, I_{V}^{0}\right)=\left(\frac{\Lambda_{H}}{\mu_{H}}, 0,0,0, \frac{\Lambda_{V}}{\mu_{V}}, 0,0,0\right)
$$

\subsubsection{The Basic Reproduction Number $R_{0}$ of the SEIR-SEI Model of Malaria Transmission}

An important concept of Epidemiological models is the basic reproduction number which is usually denoted by $R_{0}$, this number is the average number of secondary infections in the $E_{H}(\mathrm{t})$ compartment, infected by an infectious individual in the $I_{H}(\mathrm{t})$ compartment in a completely susceptible population. The Reproduction number in this model would be calculated using the Next generation matrix method. Since our model is a vector-Host model, we define the Next generation as a square matrix ' $G$ ' in which the individual of type $j$ which accounts for the infection using the reproduction number assuming that the population of type $\mathrm{i}$ is susceptible $[21,6]$.

The assumption that the population is susceptible implies that the reproduction number would be computed at DFE point. Since there are two classes of population, we have the $2 \times 2$ matrix

$$
G=\left(\begin{array}{ll}
g_{11} & g_{12} \\
g_{21} & g_{22}
\end{array}\right)=\left(\begin{array}{cc}
0 & R_{0 V} \\
R_{0 H} & 0
\end{array}\right)
$$


Let the reproduction number of the model be denoted by $R_{G}$.

From $|G-\lambda I|=0$

where $\lambda$ is an identity matrix.

$$
\begin{aligned}
& \Rightarrow|G-\lambda I|=\lambda^{2}-R_{0 H} R_{0 V}=0 \\
& \therefore \lambda=\sqrt{R_{0 H} R_{0 V}} \\
& \Rightarrow R_{G}=\sqrt{R_{0 H} R_{0 V}}
\end{aligned}
$$

From the human nonlinear system of ODEs;

$$
\begin{aligned}
& \frac{d S_{H}(t)}{d t}=\Lambda_{H}-\beta_{H} S_{H} I_{H}-\mu_{H} S_{H}+\rho R_{H} \\
& \frac{d E_{H}(t)}{d t}=\beta_{H} S_{H} I_{H}-\left(\alpha_{1}+\mu_{H}\right) E_{H} \\
& \frac{d I_{H}(t)}{d t}=\alpha_{1} E_{H}-\left(\alpha_{2}+\mu_{H}+\delta\right) I_{H}+\psi I_{H} \\
& \frac{d R_{H}(t)}{d t}=\alpha_{2} I_{H}-\left(\mu_{H}+\rho\right) R_{H}
\end{aligned}
$$

Using the next generation matrix method,

Let

$$
X=\left(E_{H}, I_{H}, S_{H}, R_{H}\right)^{T}
$$

Then

$$
X_{H}^{\prime}=\frac{d X_{H}}{d t}=\left[\begin{array}{c}
\frac{d E_{H}}{d t} \\
\frac{d I_{H}}{d t} \\
\frac{d S_{H}}{d t} \\
\frac{d R_{H}}{d t}
\end{array}\right]=\left[\begin{array}{c}
\beta_{H} S_{H} I_{H}-\left(\alpha_{1}+\mu_{H}\right) E_{H} \\
\alpha_{1} E_{H}-\left(\alpha_{2}+\mu_{H}+\delta\right) I_{H}+\psi I_{H} \\
\Lambda_{H}-\beta_{H} S_{H} I_{H}-\mu_{H} S_{H}+\rho R_{H} \\
\alpha_{2} I_{H}-\left(\mu_{H}+\rho\right) R_{H}
\end{array}\right]
$$

By splitting the matrix in the equation (27) we have; 


$$
\frac{d X_{H}}{d t}=\left[\begin{array}{c}
\beta_{H} S_{H} I_{H} \\
0 \\
0 \\
0
\end{array}\right]-\left[\begin{array}{c}
\left(\alpha_{1}+\mu_{H}\right) E_{H} \\
-\alpha_{1} E_{H}+\left(\alpha_{2}+\mu_{H}+\delta\right) I_{H}-\psi I_{H} \\
-\Lambda_{H}+\beta_{H} S_{H} I_{H}+\mu_{H} S_{H}-\rho R_{H} \\
-\alpha_{2} I_{H}+\left(\mu_{H}+\rho\right) R_{H}
\end{array}\right]
$$

This is now in the form

$$
\begin{gathered}
\frac{d X_{H}}{d t}=F_{i}(X)-V_{i}(X) \\
F_{i}(X)=\left[\begin{array}{c}
\beta_{H} S_{H} I_{H} \\
0 \\
0 \\
0
\end{array}\right]=\left[\begin{array}{c}
F_{1} \\
F_{2} \\
F_{3} \\
F_{4}
\end{array}\right] ; V_{i}(X)=\left[\begin{array}{c}
\left(\alpha_{1}+\mu_{H}\right) E_{H} \\
-\alpha_{1} E_{H}+\left(\alpha_{2}+\mu_{H}+\delta\right) I_{H}-\psi I_{H} \\
-\Lambda_{H}+\beta_{H} S_{H} I_{H}+\mu_{H} S_{H}-\rho R_{H} \\
-\alpha_{2} I_{H}+\left(\mu_{H}+\rho\right) R_{H}
\end{array}\right]=\left[\begin{array}{c}
V_{1} \\
V_{2} \\
V_{3} \\
V_{4}
\end{array}\right]
\end{gathered}
$$

Where $F_{i}(X)$ is the matrix of new infections and $V_{i}(X)$ is the matrix of other transfer terms [6]

The next step here is to linearize the matrix $F_{i}(X)$ and $V_{i}(X)$ by taking the jacobian of each term in the matrices at Disease free equilibrium point .

Let $J\left[F_{i}(X)\right]=F_{H}$ and $J\left[V_{i}(X)\right]=V_{H}$

$$
\begin{gathered}
\therefore F_{H}=\frac{\partial F_{i}(X)}{\partial X_{j}}, V_{H}=\frac{\partial V_{i}(X)}{\partial X_{j}} \quad \text { At DFE } F_{H}\left(E^{0}\right)=\frac{\partial F_{i}\left(E^{0}\right)}{\partial X_{j}}, V_{H}\left(E^{0}\right)=\frac{\partial V_{i}\left(E^{0}\right)}{\partial X_{j}} \\
\left.\frac{\partial F_{i}\left(E^{0}\right)}{\partial X_{j}}=\left(\begin{array}{llll}
\frac{\partial F_{1}\left(E^{0}\right)}{\partial E_{H}} & \frac{\partial F_{1}\left(E^{0}\right)}{\partial I_{H}} & \frac{\partial F_{1}\left(E^{0}\right)}{\partial S_{H}} & \frac{\partial F_{1}\left(E^{0}\right)}{\partial R_{H}} \\
\frac{\partial F_{2}\left(E^{0}\right)}{\partial E_{H}} & \frac{\partial F_{2}\left(E^{0}\right)}{\partial I_{H}} & \frac{\partial F_{2}\left(E^{0}\right)}{\partial S_{H}} & \frac{\partial F_{2}\left(E^{0}\right)}{\partial R_{H}} \\
\frac{\partial F_{3}\left(E^{0}\right)}{\partial E_{H}} & \frac{\partial F_{3}\left(E^{0}\right)}{\partial I_{H}} & \frac{\partial F_{3}\left(E^{0}\right)}{\partial S_{H}} & \frac{\partial F_{3}\left(E^{0}\right)}{\partial R_{H}} \\
\frac{\partial F_{4}\left(E^{0}\right)}{\partial E_{H}} & \frac{\partial F_{4}\left(E^{0}\right)}{\partial I_{H}} & \frac{\partial F_{4}\left(E^{0}\right)}{\partial S_{H}} & \frac{\partial F_{4}\left(E^{0}\right)}{\partial R_{H}}
\end{array}\right) ; \begin{array}{llll}
\frac{\partial V_{1}\left(E^{0}\right)}{\partial E_{H}} & \frac{\partial V_{1}\left(E^{0}\right)}{\partial I_{H}} & \frac{\partial V_{1}\left(E^{0}\right)}{\partial S_{H}} & \frac{\partial V_{1}\left(E^{0}\right)}{\partial R_{H}} \\
\frac{\partial V_{2}\left(E^{0}\right)}{\partial E_{H}} & \frac{\partial V_{2}\left(E^{0}\right)}{\partial I_{H}} & \frac{\partial V_{2}\left(E^{0}\right)}{\partial S_{H}} & \frac{\partial V_{2}\left(E^{0}\right)}{\partial R_{H}} \\
\frac{\partial V_{3}\left(E^{0}\right)}{\partial E_{H}} & \frac{\partial V_{3}\left(E^{0}\right)}{\partial I_{H}} & \frac{\partial V_{3}\left(E^{0}\right)}{\partial S_{H}} & \frac{\partial V_{3}\left(E^{0}\right)}{\partial R_{H}} \\
\frac{\partial V_{4}\left(E^{0}\right)}{\partial E_{H}} & \frac{\partial V_{4}\left(E^{0}\right)}{\partial I_{H}} & \frac{\partial V_{4}\left(E^{0}\right)}{\partial S_{H}} & \frac{\partial V_{4}\left(E^{0}\right)}{\partial R_{H}}
\end{array}\right)
\end{gathered}
$$

For the Reproduction number, we only need terms in the Exposed and the infected compartments [27].

Then we have the matrix 


$$
F_{H}=\left(\begin{array}{cc}
0 & \beta_{H} \frac{\Lambda_{H}}{\mu_{H}} \\
0 & 0
\end{array}\right) ; V_{H}=\left(\begin{array}{cc}
\left(\alpha_{1}+\mu_{H}\right) & 0 \\
-\alpha_{1} & \left(\alpha_{2}+\mu_{H}+\delta-\psi\right)
\end{array}\right)
$$

$R_{0 H}$ Is the spectral radius or dominant Eigen value of $\left(F_{H} V_{H}^{-1}\right)$ that is $\left|\left(F_{H} V_{H}^{-1}\right)-\lambda I\right|=0$; I is an identity matrix.

By computing the spectral radius, the reproduction number is given as;

$$
R_{0 H}=\frac{\alpha_{1} \beta_{H} \Lambda_{H}}{\mu_{H}\left(\alpha_{1}+\mu_{H}\right)\left(\alpha_{2}+\mu_{H}+\delta-\psi\right)}
$$

Similarly, by considering the nonlinear system in the Mosquito's model

$$
\begin{aligned}
& \frac{d S_{V}(t)}{d t}=\Lambda_{V}-\beta_{V} S_{V} I_{V}-\mu_{V} S_{V} \\
& \frac{d E_{V}(t)}{d t}=\beta_{V} S_{V} I_{V}-\left(\alpha_{3}+\mu_{V}\right) E_{V} \\
& \frac{d I_{V}(t)}{d t}=\alpha_{3} E_{V}-\mu_{V} I_{V}
\end{aligned}
$$

Similarly, using the Next generation matrix approach on the vectors system of equations above we have the Mosquito's reproduction number as

$$
R_{0 V}=\frac{\alpha_{3} \beta_{V} \Lambda_{V}}{\mu_{V}\left(\alpha_{3}+\mu_{V}\right)}
$$

From equation (25) we have that $R_{G}=\sqrt{R_{0 H} R_{0 V}}$ then by putting the equation (30) and (31) into (25) we have the general reproduction number of the SEIR-SEI system as:

$$
R_{G}=\sqrt{\frac{\alpha_{1} \alpha_{3} \beta_{H} \beta_{V} \Lambda_{H} \Lambda_{V}}{\mu_{H} \mu_{V}^{2}\left(\alpha_{1}+\mu_{H}\right)\left(\alpha_{3}+\mu_{V}\right)\left(\alpha_{2}+\mu_{H}+\delta-\psi\right)}}
$$

This gives the reproduction number of the complete system By alternative notations, if we let

$$
\begin{aligned}
& \left(\alpha_{1}+\mu_{H}\right)=\xi_{H} \\
& \left(\alpha_{3}+\mu_{V}\right)=\xi_{V} \\
& \left(\alpha_{2}+\mu_{H}+\delta-\psi\right)=\gamma_{H}
\end{aligned}
$$


Then,

$$
\begin{aligned}
& R_{G}=\sqrt{\frac{\alpha_{1} \alpha_{3} \beta_{H} \beta_{V} \Lambda_{H} \Lambda_{V}}{\mu_{H} \mu_{V}{ }^{2} \xi_{H} \xi_{V} \gamma_{H}}} \\
& \Rightarrow R_{G}^{2}=\frac{\alpha_{1} \alpha_{3} \beta_{H} \beta_{V} \Lambda_{H} \Lambda_{V}}{\mu_{H} \mu_{V}{ }^{2} \xi_{H} \xi_{V} \gamma_{H}}
\end{aligned}
$$

\subsection{Existence of the Endemic Equilibrium Points}

The SEI-SEI model of Malaria transmission possesses an endemic equilibrium point

$$
E^{*}=\left(S_{H}^{*}, \mathrm{E}_{H}^{*}, \mathrm{I}_{H}^{*}, \mathrm{R}_{H}^{*}, S_{V}^{*}, \mathrm{E}_{V}^{*}, \mathrm{I}_{V}^{*}\right)
$$

At this point, there is persistence of the disease in the system and hence an epidemic outbreak.

At equilibrium,

$$
\frac{d S_{H}(t)}{d t}=\frac{d E_{H}(t)}{d t}=\frac{d I_{H}(t)}{d t}=\frac{d R_{H}(t)}{d t}=\frac{d S_{V}(t)}{d t}=\frac{d E_{V}(t)}{d t}=\frac{d I_{V}(t)}{d t}=0
$$

Then,

$$
\begin{aligned}
& \Lambda_{H}-\beta_{H} S_{H}^{*} I_{H}^{*}-\mu_{H} S_{H}^{*}+\rho R_{H}^{*}=0 \\
& \beta_{H} S_{H}^{*} I_{H}^{*}-\left(\alpha_{1}+\mu_{H}\right) E_{H}^{*}=0 \\
& \alpha_{1} E_{H}^{*}-\left(\alpha_{2}+\mu_{H}+\delta-\psi\right) I_{H}^{*}=0 \\
& \alpha_{1} I_{H}^{*}-\left(\mu_{H}+\rho\right) R_{H}^{*}=0 \\
& \Lambda_{V}-\beta_{V} S_{V}^{*} I_{V}^{*}-\mu_{V} S_{V}^{*}=0 \\
& \beta_{V} S_{V}^{*} I_{V}^{*}-\left(\alpha_{1}+\mu_{V}\right) E_{V}^{*}=0 \\
& \alpha_{3} E_{V}^{*}-\mu_{V} I_{V}^{*}=0
\end{aligned}
$$

We solve the system of equation (36) simultaneously for the corresponding endemic point

s. From $0=\alpha_{1} E_{H}^{*}-\left(\alpha_{2}+\mu_{H}+\delta\right) I_{H}^{*}+\psi I_{H}^{*}$ in the system, we can write that $\alpha_{1} E_{H}^{*}=\left(\alpha_{2}+\mu_{H}+\delta-\psi\right) I_{H}^{*}$ Thus we have

$$
E_{H}^{*}=\frac{\left(\alpha_{2}+\mu_{H}+\delta-\psi\right) I_{H}^{*}}{\alpha_{1}}
$$


Put (37) into $\frac{d S_{H}(\mathrm{t})}{d t}$ we have the relation,

$$
\beta_{H} S_{H} I_{H}-\frac{\left(\alpha_{1}+\mu_{H}\right)\left(\alpha_{2}+\mu_{H}+\delta-\psi\right) I_{H}}{\alpha_{1}}=0
$$

This implies that

$$
\begin{aligned}
& I_{H}\left[\beta_{H} S_{H}-\frac{\left(\alpha_{1}+\mu_{H}\right)\left(\alpha_{2}+\mu_{H}+\delta-\psi\right)}{\alpha_{1}}\right]=0 \text { Where } I_{H} \neq 0 \\
& \Rightarrow \beta_{H} S_{H}-\frac{\left(\alpha_{1}+\mu_{H}\right)\left(\alpha_{2}+\mu_{H}+\delta-\psi\right)}{\alpha_{1}}=0 ; \\
& S_{H}^{*}=\frac{\left(\alpha_{1}+\mu_{H}\right)\left(\alpha_{2}+\mu_{H}+\delta-\psi\right)}{\beta_{H} \alpha_{1}}
\end{aligned}
$$

Again from $\frac{d R_{H}(\mathrm{t})}{d t}$, we have

$$
R_{H}^{*}=\frac{\alpha_{2} I_{H}^{*}}{\left(\mu_{H}+\rho\right)}
$$

By substituting (39) and (40) into $\frac{d S_{H}(\mathrm{t})}{d t}$ and solving accordingly we have;

$I_{H}^{*}=\frac{\alpha_{1} \beta_{H} \Lambda_{H}\left(\mu_{H}+\rho\right)-\mu_{H}\left(\mu_{H}+\rho\right)\left(\alpha_{1}+\mu_{H}\right)\left(\alpha_{2}+\mu_{H}+\delta-\psi\right)}{\beta_{H}\left(\mu_{H}+\rho\right)\left(\alpha_{1}+\mu_{H}\right)\left(\alpha_{2}+\mu_{H}+\delta-\psi\right)-\beta_{H} \rho \alpha_{1} \alpha_{2}}$

Similarly by solving the system (36) appropriately, we obtain the endemic point

$$
\begin{gathered}
E_{H}^{*}=\frac{\left(\alpha_{2}+\mu_{H}+\delta-\psi\right)\left[\alpha_{1} \beta_{H} \Lambda_{H}\left(\mu_{H}+\rho\right)-\mu_{H}\left(\mu_{H}+\rho\right)\left(\alpha_{1}+\mu_{H}\right)\left(\alpha_{2}+\mu_{H}+\delta-\psi\right)\right]}{\alpha_{1} \beta_{H}\left[\left(\mu_{H}+\rho\right)\left(\alpha_{1}+\mu_{H}\right)\left(\alpha_{2}+\mu_{H}+\delta-\psi\right)-\rho \alpha_{1} \alpha_{2}\right]} \\
R_{H}^{*}=\frac{\alpha_{2} \alpha_{1} \beta_{H} \Lambda_{H}-\mu_{H} \alpha_{2}\left(\alpha_{1}+\mu_{H}\right)\left(\alpha_{2}+\mu_{H}+\delta-\psi\right)}{\beta_{H}\left[\left(\mu_{H}+\rho\right)\left(\alpha_{2}+\mu_{H}\right)\left(\alpha_{2}+\mu_{H}+\delta-\psi\right)-\alpha_{1} \alpha_{2} \rho\right]} \\
S_{V}^{*}=\frac{\mu_{V}\left(\alpha_{3}+\mu_{V}\right)}{\beta_{V} \alpha_{3}} \\
E_{V}^{*}=\frac{\alpha_{3} \beta_{V} \Lambda_{V}-\mu_{V}^{2}\left(\alpha_{3}+\mu_{V}\right)}{\alpha_{3} \beta_{V}\left(\alpha_{3}+\mu_{V}\right)} \\
I_{V}^{*}=\frac{\Lambda_{V} \beta_{V} \alpha_{3}-\mu_{V}^{2}\left(\alpha_{3}+\mu_{V}\right)}{\mu_{V} \beta_{V}\left(\alpha_{3}+\mu_{V}\right)}
\end{gathered}
$$




\subsection{Stability of the Disease-Free Equilibrium}

We now check for the stability of the model at DFE by taking the jacobian of the seven dimensional ODES in equation (1) and obtaining its corresponding Eigen values.

The SEIR-SEI is stable if all of the Eigen values obtained from the linearized system are negative real values.

We have the jacobian of the model to be given as:

$J\left(S_{H}, E_{H}, I_{H}, R_{H}, S_{V}, E_{V}, I_{V}\right)$

$$
=\left(\begin{array}{cccrcccc}
-\beta_{H} I_{H}-\mu_{H} & 0 & -\beta_{H} S_{H} & \rho & 0 & 0 & 0 & \\
\beta_{H} I_{H} & -\left(\alpha_{1}+\mu_{H}\right) & \beta_{H} S_{H} & 0 & 0 & 0 & 0 & \\
0 & \alpha_{1} & -\left(\alpha_{2}+\mu_{H}+\delta-\psi\right) & 0 & 0 & 0 & 0 & \\
0 & 0 & \alpha_{2} & -\left(\mu_{H}+\rho\right) & 0 & 0 & 0 \\
0 & 0 & 0 & 0 & -\beta_{V} I_{V}-\mu_{V} & 0 & 0 \\
0 & 0 & 0 & 0 & \beta_{V} I_{V} & -\left(\alpha_{3}+\mu_{V}\right) & \beta_{V} S_{V} \\
0 & 0 & 0 & 0 & 0 & \alpha_{3} & -\mu_{V} &
\end{array}\right)
$$

At Disease-Free equilibrium point,

$$
J\left(E^{0}\right)=\left(\begin{array}{cccccccc}
-\mu_{H} & 0 & -\beta_{H} \frac{\Lambda_{H}}{\mu_{H}} & \rho & 0 & 0 & 0 & \\
0 & -\left(\alpha_{1}+\mu_{H}\right) & \beta_{H} \frac{\Lambda_{H}}{\mu_{H}} & 0 & 0 & 0 & 0 & \\
0 & \alpha_{1} & -\left(\alpha_{2}+\mu_{H}+\delta-\psi\right) & 0 & 0 & 0 & 0 & \\
0 & 0 & \alpha_{2} & -\left(\mu_{H}+\rho\right) & 0 & 0 & 0 \\
0 & 0 & 0 & 0 & -\mu_{V} & 0 & -\beta_{V} \frac{\Lambda_{V}}{\mu_{V}} \\
0 & 0 & 0 & 0 & 0 & -\left(\alpha_{3}+\mu_{V}\right) & \beta_{V} \frac{\Lambda_{V}}{\mu_{V}} \\
0 & 0 & 0 & 0 & 0 & \alpha_{3} & -\mu_{V}
\end{array}\right)
$$

By inserting our alternative notation

Let 


$$
\begin{array}{ll}
\left(\alpha_{1}+\mu_{H}\right)=\xi_{H} ; & K_{1}=\beta_{H} \frac{\Lambda_{H}}{\mu_{H}} \\
\left(\alpha_{3}+\mu_{V}\right)=\xi_{V} ; & K_{2}=\beta_{V} \frac{\Lambda_{V}}{\mu_{V}} \\
\left(\alpha_{2}+\mu_{H}+\delta-\psi\right)=\gamma_{H} ; &
\end{array}
$$

We have,

$$
J\left(E^{0}\right)=\left(\begin{array}{cccccccc}
-\mu_{H} & 0 & -K_{1} & \rho & 0 & 0 & 0 \\
0 & -\xi_{H} & K_{1} & 0 & 0 & 0 & 0 \\
0 & \alpha_{1} & -\gamma_{H} & 0 & 0 & 0 & 0 \\
0 & 0 & \alpha_{2} & -\varepsilon_{H} & 0 & 0 & 0 \\
0 & 0 & 0 & 0 & -\mu_{V} & 0 & -K_{2} \\
0 & 0 & 0 & 0 & 0 & -\xi_{V} & K_{2} \\
0 & 0 & 0 & 0 & 0 & \alpha_{3} & -\mu_{V}
\end{array}\right)
$$

For the Eigen-values of the matrix,

$$
\left|J\left(E^{0}\right)-\lambda I\right|=\left(\begin{array}{cccccccc}
-\mu_{H}-\lambda & 0 & -K_{1} & \rho & 0 & 0 & 0 \\
0 & -\xi_{H}-\lambda & K_{1} & 0 & 0 & 0 & 0 & \\
0 & \alpha_{1} & -\gamma_{H}-\lambda & 0 & 0 & 0 & 0 & \\
0 & 0 & \alpha_{2} & -\varepsilon_{H}-\lambda & 0 & 0 & 0 \\
0 & 0 & 0 & 0 & -\mu_{V}-\lambda & 0 & -K_{2} \\
0 & 0 & 0 & 0 & 0 & -\xi_{V}-\lambda & K_{2} \\
0 & 0 & 0 & 0 & 0 & \alpha_{3} & -\mu_{V}-\lambda
\end{array}\right)
$$

Now by applying some matrix techniques on equation (51), it is clear that the first column of (51) contains a diagonal term ' $-\mu_{H}-\lambda$ ' only. Hence, $\lambda_{1}=-\mu_{H}$ and we eliminate the first row and column in (51) to have a new matrix $J_{0}\left(E^{0}\right)$

$$
J_{0}\left(E^{0}\right)\left(\begin{array}{cccrrrr}
-\xi_{H}-\lambda & K_{1} & 0 & 0 & 0 & 0 \\
\alpha_{1} & -\gamma_{H}-\lambda & 0 & 0 & 0 & 0 \\
0 & \alpha_{2} & -\varepsilon_{H}-\lambda & 0 & 0 & 0 \\
0 & 0 & 0 & -\mu_{V}-\lambda & 0 & -K_{2} \\
0 & 0 & 0 & 0 & -\xi_{V}-\lambda & K_{2} \\
0 & 0 & 0 & 0 & \alpha_{3} & -\mu_{V}-\lambda
\end{array}\right)
$$


It also clear from (52) that the third and fourth column contains only diagonal terms ' $-\mu_{V}-\lambda$ ' and ' $-\varepsilon_{H}-\lambda$ ' which produces two Eigen values $\lambda_{2}=-\mu_{V}$ and $\lambda_{3}=-\varepsilon_{H}$. Hence we eliminate the third and fourth rows and columns so as to have a new matrix $J_{1}\left(E^{0}\right)$.

$$
\begin{gathered}
\therefore J_{1}\left(E^{0}\right)=\left(\begin{array}{cccc}
-\xi_{H}-\lambda & K_{1} & 0 & 0 \\
\alpha_{1} & -\gamma_{H}-\lambda & 0 & 0 \\
0 & 0 & -\xi_{V}-\lambda & K_{2} \\
0 & 0 & \alpha_{3} & -\mu_{V}-\lambda
\end{array}\right) \\
J_{1}\left(E^{0}\right)=\left(\begin{array}{cccc}
-\xi_{H}-\lambda & K_{1} & 0 & 0 \\
\alpha_{1} & -\gamma_{H}-\lambda & 0 & 0 \\
0 & 0 & -\xi_{V}-\lambda & K_{2} \\
0 & 0 & \alpha_{3} & -\mu_{V}-\lambda
\end{array}\right)=\left(\begin{array}{cccc}
-\xi_{H} & K_{1} & 0 & 0 \\
\alpha_{1} & -\gamma_{H} & 0 & 0 \\
0 & 0 & -\xi_{V} & K_{2} \\
0 & 0 & \alpha_{3} & -\mu_{V}
\end{array}\right)-\left(\begin{array}{cccc}
\lambda & 0 & 0 & 0 \\
0 & \lambda & 0 & 0 \\
0 & 0 & \lambda & 0 \\
0 & 0 & 0 & \lambda
\end{array}\right)
\end{gathered}
$$

Let

$$
\begin{gathered}
J_{1 A}\left(E^{0}\right)=\left(\begin{array}{cccc}
-\xi_{H} & K_{1} & 0 & 0 \\
\alpha_{1} & -\gamma_{H} & 0 & 0 \\
0 & 0 & -\xi_{V} & K_{2} \\
0 & 0 & \alpha_{3} & -\mu_{V}
\end{array}\right) \\
\therefore J_{1}\left(\mathrm{E}^{0}\right)=\left|\mathrm{J}_{1 A}\left(E^{0}\right)-\lambda \mathrm{I}\right|
\end{gathered}
$$

By performing the following row transformation (Gaussian elimination method) on the matrix (55) with the operations,

$$
\begin{aligned}
& R_{4}^{*}=R_{4}+\frac{\alpha_{3}}{\xi_{V}} R_{3} \\
& R_{2}^{*}=R_{2}+\frac{\alpha_{1}}{\xi_{H}} R_{1}
\end{aligned}
$$

We have the new Jacobian matrix

$$
J_{1 A}\left(E^{0}\right)=\left(\begin{array}{cccc}
-\xi_{H} & K_{1} & 0 & 0 \\
0 & -\gamma_{H}+\frac{\alpha_{1} K_{1}}{\xi_{H}} & 0 & 0 \\
0 & 0 & -\xi_{V} & K_{2} \\
0 & 0 & 0 & -\mu_{V}+\frac{\alpha_{3} K_{2}}{\xi_{V}}
\end{array}\right)
$$

For the Eigen values, 


$$
\therefore J_{1}\left(E^{0}\right)=\left(\begin{array}{cccc}
-\xi_{H}-\lambda & K_{1} & 0 & 0 \\
0 & \left(-\gamma_{H}+\frac{\alpha_{1} K_{1}}{\xi_{H}}\right)-\lambda & 0 & 0 \\
0 & 0 & -\xi_{V}-\lambda & K_{2} \\
0 & 0 & 0 & \left(-\mu_{V}+\frac{\alpha_{3} K_{2}}{\xi_{V}}\right)-\lambda
\end{array}\right)
$$

By applying the same matrix techniques which was applied on (51) accordingly and replacing alternative notations, we have the 7 Eigen values for the model as:

$$
\begin{aligned}
& \lambda_{1}=-\mu_{H}, \lambda_{2}=-\mu_{V}, \lambda_{3}=-\left(\mu_{H}+\rho\right), \lambda_{4}=-\left(\alpha_{1}+\mu_{H}\right), \lambda_{5}=-\left(\alpha_{3}+\mu_{V}\right), \\
& \lambda_{6}=-\left(\gamma_{H}-\frac{\alpha_{1} \beta_{H} \Lambda_{H}}{\xi_{H} \mu_{H}}\right), \lambda_{7}=-\left(\mu_{V}-\frac{\alpha_{3} \beta_{V} \Lambda_{V}}{\xi_{V} \mu_{V}}\right)
\end{aligned}
$$

Clearly all our Eigen values are negative real values, and then the Disease-free equilibrium (DFE) is stable.

\subsubsection{Theorem 2:}

The Disease Free equilibrium (DFE) is locally asymptotically stable if $R_{G}<1$.

\section{Proof:}

From the Eigen-values $\lambda_{6}$ and $\lambda_{7}$

$$
\begin{aligned}
& \lambda_{6}=-\left(\gamma_{H}-\frac{\alpha_{1} \beta_{H} \Lambda_{H}}{\xi_{H} \mu_{H}}\right)=-\gamma_{H}\left(1-\frac{\alpha_{1} \beta_{H} \Lambda_{H}}{\xi_{H} \mu_{H} \gamma_{H}}\right) \\
& \lambda_{7}=-\left(\mu_{V}-\frac{\alpha_{3} \beta_{V} \Lambda_{V}}{\xi_{V} \mu_{V}}\right)=-\mu_{V}\left(1-\frac{\alpha_{3} \beta_{V} \Lambda_{V}}{\xi_{V} \mu_{V}^{2}}\right)
\end{aligned}
$$

Since $\frac{\alpha_{1} \beta_{H} \Lambda_{H}}{\xi_{H} \mu_{H} \gamma_{H}}=R_{0 H} ; \frac{\alpha_{3} \beta_{V} \Lambda_{V}}{\xi_{V} \mu_{V}^{2}}=R_{0 V}$

Then,

$$
\lambda_{6}=-\gamma_{H}\left(1-\frac{\alpha_{1} \beta_{H} \Lambda_{H}}{\xi_{H} \mu_{H} \gamma_{H}}\right)=-\gamma_{H}\left(1-R_{0 H}\right)
$$




$$
\lambda_{7}=-\mu_{V}\left(1-\frac{\alpha_{3} \beta_{V} \Lambda_{V}}{\xi_{V} \mu_{V}^{2}}\right)=-\mu_{V}\left(1-R_{0 V}\right)
$$

Equation (62) and (63) above holds if and only if $R_{0 H}<1$ and $R_{0 V}<1$ holds respectively.

Thus, the DFE is stable if $R_{0 H}<1, R_{0 V}<1$.

From equation (26), $R_{G}=\sqrt{R_{0 H} R_{0 V}}$, which implies that $R_{G}^{2}=R_{0 H} R_{0 V}$.

Then if

$$
\begin{aligned}
& R_{0 H}<1, R_{0 V}<1 \\
& \therefore R_{G}^{2}=R_{0 H} R_{0 V}<1 \\
& \Rightarrow R_{G}<\sqrt{1}
\end{aligned}
$$

Showing that $R_{G}<1$ holds

This proves theorem 2 .

\subsection{Stability of the Endemic Equilibrium Point}

We evaluate $\left|J\left(E_{H}^{*}\right)-\lambda I\right|=0,\left|J\left(E_{V}^{*}\right)-\lambda I\right|=0$ for the Host and Vector respectively.

From the Human 4-dimensional differential equations we have the Jacobian as:

$$
J\left(S_{H}, E_{H}, I_{H}, R_{H}\right)=\left(\begin{array}{cccc}
-\beta_{H} I_{H}-\mu_{H} & 0 & -\beta_{H} S_{H} & -\rho \\
\beta_{H} I_{H} & -\left(\alpha_{1}+\mu_{H}\right) & \beta_{H} S_{H} & 0 \\
0 & \alpha_{1} & -\left(\alpha_{2}+\mu_{H}+\delta-\psi\right) & 0 \\
0 & 0 & \alpha_{2} & -\left(\mu_{H}+\rho\right)
\end{array}\right)
$$

At Endemic points 


$$
J\left(S_{H}^{*}, E_{H}^{*}, I_{H}^{*}, R_{H}^{*}\right)=\left(\begin{array}{cccc}
-\beta_{H} I_{H}^{*}-\mu_{H} & 0 & -\beta_{H} S_{H}^{*} & -\rho \\
\beta_{H} I_{H}^{*} & -\left(\alpha_{1}+\mu_{H}\right) & \beta_{H} S_{H}^{*} & 0 \\
0 & \alpha_{1} & -\left(\alpha_{2}+\mu_{H}+\delta-\psi\right) & 0 \\
0 & 0 & \alpha_{2} & -\left(\mu_{H}+\rho\right)
\end{array}\right)
$$

By substituting equation (35) and alternative notations in (49)

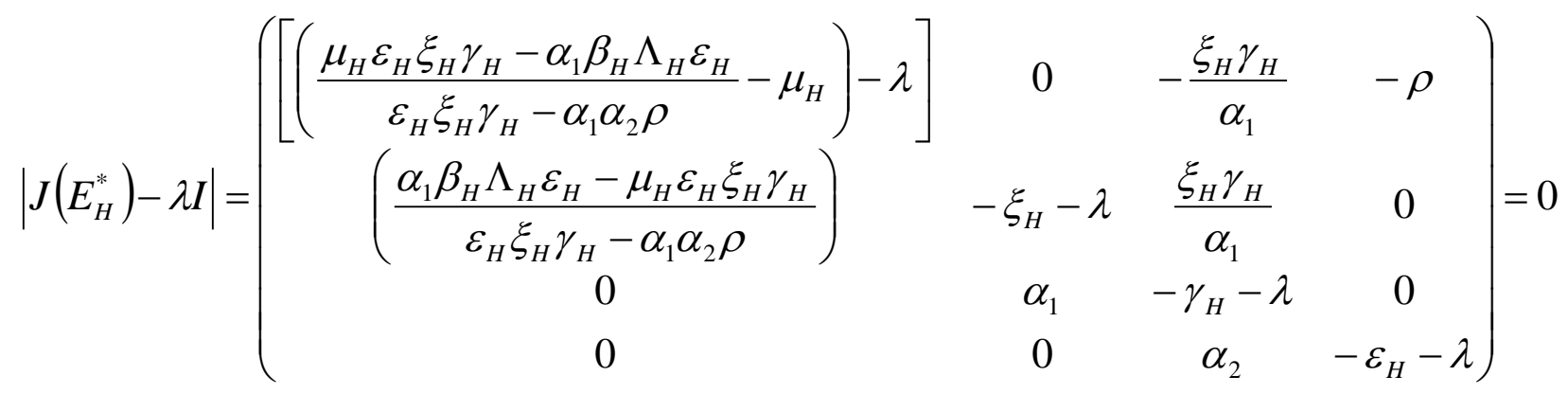

The Characteristic equation for the above matrix gives

$$
\left|J\left(E_{H}^{*}\right)-\lambda I\right|=Q_{4} \lambda^{4}+Q_{3} \lambda^{3}+Q_{2} \lambda^{2}+Q_{1} \lambda+Q_{0}
$$

Where

$$
\begin{aligned}
& Q_{4}=1 ; \\
& Q_{3}=\left[\left(\varepsilon_{H}+\xi_{H}+\gamma_{H}\right)-\left(\frac{\mu_{H} \alpha_{1} \alpha_{1} \rho-\alpha_{1} \beta_{H} \Lambda_{H} \varepsilon_{H}}{\varepsilon_{H} \xi_{H} \gamma_{H}-\alpha_{1} \alpha_{2} \rho}\right)\right] ; \\
& Q_{2}=\left[\left(\xi_{H} \gamma_{H}+\xi_{H} \varepsilon_{H}+\gamma_{H} \varepsilon_{H}+\xi_{H} \gamma_{H}\right)-\left(\varepsilon_{H}+\xi_{H}+\gamma_{H}\right)\left(\frac{\mu_{H} \alpha_{1} \alpha_{1} \rho-\alpha_{1} \beta_{H} \Lambda_{H} \varepsilon_{H}}{\varepsilon_{H} \xi_{H} \gamma_{H}-\alpha_{1} \alpha_{2} \rho}\right)\right] \\
& Q_{1}=\left(\frac{\xi_{H} \gamma_{H} \alpha_{1} \beta_{H} \Lambda_{H} \varepsilon_{H}-\mu_{H} \varepsilon_{H} \xi_{H}^{2} \gamma_{H}^{2}}{\varepsilon_{H} \xi_{H} \gamma_{H}-\alpha_{1} \alpha_{2} \rho}\right)-\left(\xi_{H} \gamma_{H}+\xi_{H} \varepsilon_{H}+\gamma_{H} \varepsilon_{H}+\xi_{H} \gamma_{H}\right)\left(\frac{\mu_{H} \alpha_{1} \alpha_{1} \rho-\alpha_{1} \beta_{H} \Lambda_{H} \varepsilon_{H}}{\varepsilon_{H} \xi_{H} \gamma_{H}-\alpha_{1} \alpha_{2} \rho}\right) \\
& Q_{0}=\left[\frac{\xi_{H} \gamma_{H} \alpha_{1} \beta_{H} \Lambda_{H} \varepsilon_{H}^{2}-\mu_{H} \varepsilon_{H}^{2} \xi_{H}^{2} \gamma_{H}^{2}}{\varepsilon_{H} \xi_{H} \gamma_{H}-\alpha_{1} \alpha_{2} \rho} \frac{\rho \alpha_{1} \alpha_{2} \beta_{H} \Lambda_{H} \varepsilon_{H}-\rho \mu_{H} \varepsilon_{H} \xi_{H} \gamma_{H} \alpha_{1} \alpha_{2}}{\varepsilon_{H} \xi_{H} \gamma_{H}-\alpha_{1} \alpha_{2} \rho}\right]
\end{aligned}
$$

Using the Routh-Hurwitz criterion for quartic polynomials, the characteristic equation (65) yields negative Eigen-values (stable) if and only if: $Q_{4}>0, Q_{3}>0, Q_{2}>0, Q_{1}>0, Q_{0}>0$ holds and this clearly seen. Also from this criterion, the characteristic equation has negative eigen values 
(Stable)

if $\quad$ the inequalities:

$$
Q_{3} Q_{2}-Q_{1}>0, Q_{3} Q_{2} Q_{1}-Q_{1}^{2}-Q_{3}^{2} Q_{0}>0, Q_{3} Q_{2} Q_{1} Q_{0}-Q_{4} Q_{1}^{2} Q_{0}>0 \text { are }
$$

satisfied. This is trivial as well and has been verified; hence the endemic equilibrium is stable and we can hence have the Lemma.

\subsubsection{Lemma 3:}

The endemic equilibrium $E_{H}^{*}$ of the SEIR-SEI vector host malaria transmission model is locally asymptotically stable if the inequalities $Q_{3} Q_{2}-Q_{1}>0, Q_{3} Q_{2} Q_{1}-Q_{1}^{2}-Q_{3}^{2} Q_{0}>0, Q_{3} Q_{2} Q_{1} Q_{0}-Q_{4} Q_{1}^{2} Q_{0}>0$ are satisfied

\subsubsection{Lemma 4:}

The positive equilibrium $E_{H}^{*}$ of Human is locally asymptotically stable if $R_{G}>1$.

\section{Proof:}

Using the Routh- Hurwitz criterion in the Characteristic polynomial in equation (2), we have that $Q_{4}>0, Q_{3}>0, Q_{2}>0, Q_{1}>0, Q_{0}>0$ must hold.

Here,

$$
Q_{0}=\left[\frac{\xi_{H} \gamma_{H} \alpha_{1} \beta_{H} \Lambda_{H} \varepsilon_{H}^{2}-\mu_{H} \varepsilon_{H}^{2} \xi_{H}^{2} \gamma_{H}^{2}}{\varepsilon_{H} \xi_{H} \gamma_{H}-\alpha_{1} \alpha_{2} \rho}+\frac{\rho \alpha_{1} \alpha_{2} \beta_{H} \Lambda_{H} \varepsilon_{H}-\rho \mu_{H} \varepsilon_{H} \xi_{H} \gamma_{H} \alpha_{1} \alpha_{2}}{\varepsilon_{H} \xi_{H} \gamma_{H}-\alpha_{1} \alpha_{2} \rho}\right]
$$

By mathematical manipulations of $Q_{0}$ in terms of the human reproduction number, we have

$$
\begin{aligned}
& Q_{0}=\left[\frac{\mu_{H} \varepsilon_{H}^{2} \xi_{H}^{2} \gamma_{H}^{2}+\alpha_{1} \alpha_{2} \rho \varepsilon_{H} \xi_{H} \gamma_{H} \mu_{H}}{\varepsilon_{H} \xi_{H} \gamma_{H}-\alpha_{1} \alpha_{2} \rho}\right]\left(\frac{\alpha_{1} \beta_{H} \Lambda_{H}}{\mu_{H} \xi_{H} \gamma_{H}}-1\right) \\
& \therefore Q_{0}=\left[\frac{\mu_{H} \varepsilon_{H}^{2} \xi_{H}^{2} \gamma_{H}^{2}+\alpha_{1} \alpha_{2} \rho \varepsilon_{H} \xi_{H} \gamma_{H} \mu_{H}}{\varepsilon_{H} \xi_{H} \gamma_{H}-\alpha_{1} \alpha_{2} \rho}\right]\left(R_{0 H}-1\right)>0 \\
& \Rightarrow\left[\frac{\mu_{H} \varepsilon_{H}^{2} \xi_{H}^{2} \gamma_{H}^{2}+\alpha_{1} \alpha_{2} \rho \varepsilon_{H} \xi_{H} \gamma_{H} \mu_{H}}{\varepsilon_{H} \xi_{H} \gamma_{H}-\alpha_{1} \alpha_{2} \rho}\right]\left(R_{0 H}-1\right)>0
\end{aligned}
$$


The Inequality (68) will hold if and only if $R_{0 H}>1$. This Proves the Lemma 4

Similarly for the Vector Population (Mosquitoes), considering the Vector system of differential equations

$$
\begin{aligned}
& \frac{d S_{V}(t)}{d t}=\Lambda_{V}-\beta_{V} S_{V} I_{V}-\mu_{V} S_{V} \\
& \frac{d E_{V}(t)}{d t}=\beta_{V} S_{V} I_{V}-\left(\alpha_{3}+\mu_{V}\right) E_{V} \\
& \frac{d I_{V}(t)}{d t}=\alpha_{3} E_{V}-\mu_{V} I_{V}
\end{aligned}
$$

By taking the Jacobian at the Endemic point $E_{V}^{*}=\left(S_{V}^{*}, E_{V}^{*}, I_{V}^{*}\right)$ we have;

$$
\left|J\left(E_{V}^{*}\right)-\lambda I\right|=\left(\begin{array}{ccc}
\left(\frac{\mu_{V}^{2} \xi_{V}-\alpha_{3} \beta_{V} \Lambda_{V}}{\mu_{V} \xi_{V}}-\mu_{V}\right)-\lambda & 0 & -\beta_{V} S_{V}^{*} \\
\frac{\alpha_{3} \beta_{V} \Lambda_{V}-\mu_{V}^{2} \xi_{V}}{\mu_{V} \xi_{V}} & -\xi_{V}-\mu & \beta_{V} S_{V}^{*} \\
0 & \alpha_{3} & -\mu_{V}-\lambda
\end{array}\right)
$$

The Characteristic equation of the above matrix is given as:

$$
\left|J\left(E_{V}^{*}\right)-\lambda I\right|=A_{3} \lambda^{3}+A_{2} \lambda^{2}+A_{1} \lambda+A_{0}
$$

Where,

$$
A_{3}=1 ; A_{2}=\left(\frac{\alpha_{3} \beta_{V} \Lambda_{V}}{\mu_{V} \xi_{V}}+\left(\xi_{V}+\mu_{V}\right)\right) ; A_{1}=\left(\frac{\alpha_{3} \beta_{V} \Lambda_{V}\left(\xi_{V}+\mu_{V}\right)}{\mu_{V}}\right) ; A_{0}=\alpha_{3} \beta_{V} \Lambda_{V}-\mu_{V}^{2} \xi_{V} .
$$

It has been clearly verified using Routh-Hurwitz criterion that the system has all its eigen value to be negative if and only if $A_{2}>0, A_{0}>0, A_{2} A_{1}>A_{0}$ holds. This is very trivial and hence the Endemic equilibrium is stable.

\subsubsection{Lemma 5:}

The positive endemic equilibrium $E_{V}^{*}$ of the vector (mosquito) population is locally asymptotically stable if $R_{0 V}>1$. 


\section{Proof:}

By considering the condition for Routh-Hurwitz stability $A_{0}>0$ we have that

$$
\begin{gathered}
A_{0}=\alpha_{3} \beta_{V} \Lambda_{V}-\mu_{V}^{2} \xi_{V}>0 \\
\therefore \alpha_{3} \beta_{V} \Lambda_{V}-\mu_{V}^{2} \xi_{V}>0 \\
\Rightarrow \mu_{V}^{2} \xi_{V}\left(\frac{\alpha_{3} \beta_{V} \Lambda_{V}}{\mu_{V}^{2} \xi_{V}}-1\right)>0 ; R_{0 V}=\frac{\alpha_{3} \beta_{V} \Lambda_{V}}{\mu_{V}^{2} \xi_{V}} \\
\therefore \mu_{V}^{2} \xi_{V}\left(\frac{\alpha_{3} \beta_{V} \Lambda_{V}}{\mu_{V}^{2} \xi_{V}}-1\right)=\mu_{V}^{2} \xi_{V}\left(R_{0 V}-1\right)>0 \\
\Rightarrow \mu_{V}^{2} \xi_{V}\left(R_{0 V}-1\right)>0
\end{gathered}
$$

The inequality (5) holds if and only if $R_{0 V}>1$.

This completes the proof and hence proves the Lemma 5

\subsubsection{Theorem 3:}

The Endemic equilibrium $E_{H V}^{*}$ of the Vector-Host system (Human and Mosquito) is locally asymptotically stable if $R_{G}>1$.

\section{Proof:}

We have that $R_{0 H}>1, R_{0 V}>1$ for the Host (Human) and the Vector (Mosquito) respectively from Lemma 4 and Lemma 5.Then,

$$
\begin{aligned}
& R_{0 H}>1, R_{0 V}>1 ; \\
& \Rightarrow R_{0 H} R_{0 V}>1 ; \\
& \therefore R_{G}^{2}=R_{0 H} R_{0 V}>1 ; \\
& \Rightarrow R_{G}^{2}>1 ; R_{G}>\sqrt{1} ; \\
& \therefore R_{G}>1 .
\end{aligned}
$$

This proves the Theorem 3. 


\subsection{Semi-Analytical Solution to the Seir-Sei Vector Host Model of Malaria Transmission by Variational Iteration Method (VIM)}

The implementation of semi-analytical algorithms or methods has spontaneously developed over the years in the field of numerical analysis and computational mathematics.

Numerous researchers have implemented some methods appropriately in providing exact solutions to ordinary and partial differential equations viz [40-43]. Very recently, Loyinmi Adedapo C. and Akinfe Timilehin K. (2020) implemented an algorithm using the Elzaki transform to provide exact solutions to the Burgers-Huxley equation of three distinct cases as a result of variation in the equation parameters [44], Again in (2019), using a hybrid algorithm involving Elzaki transform and homotopy perturbation method (EHTPM), they proffered exact solution to the family of Fisher's reaction-diffusion equation which is well applicable in genetics, stochastic processes, nuclear reactor theory, and so on. See ref [49]

Nadeem M, Li F, Ahmad H (2019) solved the fourth-order parabolic partial differential equation with variable coefficients using modified Laplace variational iteration method [45] and so on [46-48]

The idea of Variational iteration method (VIM) was introduced by Ji-Huan He (1998) [29] who modified the general Lagrange multiplier proposed by Inokuti (1978) [32] to solve nonlinear problems. Abbasbandy and Shivanian (2009) [33], Abdou and Soliman (2005) [35], Mosmani and Abuasad (2006) [34] have implemented this method to solve effectively, easily and accurately, a large class of nonlinear problems with approximations which converges quickly to accurate solutions.

The implementation of asymptotic techniques/methods is quite an interesting and demanding field of computational mathematics as there is no single best method or algorithm for a 
problem/model. The suitability of an algorithm to a problem depends on the simplicity, computational stress and radius/rate of convergence of such algorithm or method.

Adedapo Loyinmi C. and Akinfe Timilehin K. and other authors have buttressed these facts with their convergence analyses [49]

\subsection{Basic Idea of VIM}

Consider a non-linear differential equation

$$
L u(t)+N u(t)=g(t)
$$

$L$ is the linear operator, $N$ is the nonlinear operator and $g(t)$ is a known analytic function. We construct a correction functional for the equation (1) which is given as:

$$
u_{n+1}(t)=u_{n}(t)+\int_{0}^{t} \pi(x)\left[L u_{n}(x)+N \tilde{u}_{n}(x)-g(x)\right] d x
$$

Where $\pi$ is a lagragian multiplier which can be obtained optimally and expressed as:

$$
\pi(x)=\frac{(-1)^{n}}{(n-1) !}(x-t)^{n-1}
$$

Where $n$ is the highest order of the differential equation.

\subsection{Solution of the Model using Variational Iteration Method}

We consider the model's system of equations in (1), Subject to the initial condition (State Variables) Adopted from [1]. We have,

$$
\begin{aligned}
& S_{H}(0)=0.83, E_{H}(0)=0.08, I_{H}(0)=0.07, R_{H}(0)=0.02 S_{V}(0)=0.7, \\
& E_{V}(0)=0.2, I_{V}(0)=0.1
\end{aligned}
$$


Table 2: Parameter description and Values of model

\begin{tabular}{|c|c|c|c|}
\hline Parameter & Description Of Parameter & Value & Source \\
\hline$\Lambda_{H}$ & Recruitment Rate of Humans & 1.2 & Osman et al.(2017) [1] \\
\hline$\Lambda_{V}$ & $\begin{array}{l}\text { Recruitment } \quad \text { Rate of } \\
\text { Mosquitoes }\end{array}$ & 0.7 & Osman et al. (2017) [1] \\
\hline$\alpha_{1}$ & $\begin{array}{l}\text { Rate of Development from } E(t) \\
\text { to } I(t) \text { for Humans }\end{array}$ & 0.05 & S. Olaniyi et al. (2013) [2] \\
\hline$\alpha_{2}$ & Recovery Rate of Humans & 0.0035 & Shah NH et al. (2013) [21] \\
\hline$\alpha_{3}$ & $\begin{array}{l}\text { Rate of Development from } E(t) \\
\text { to } I(t) \text { for Mosquitoes }\end{array}$ & 0.083 & Shah NH et al. (2013) [21] \\
\hline$\mu_{H}$ & Death Rate of Humans & 0.0115 & Estimated \\
\hline$\mu_{V}$ & Death Rate of Mosquitoes & 0.05 & Macdonald G. (1957) [5] \\
\hline$\eta_{V}$ & Mosquito Biting rate & 0.46 & Jia Li (2015) [35] \\
\hline$q_{H}$ & $\begin{array}{l}\text { Probability of transmission } \\
\text { from an infectious mosquito to } \\
\text { Human }\end{array}$ & 0.022 & Chitnis $N$ et al. (2008) [37] \\
\hline$q_{V}$ & $\begin{array}{l}\text { Probability of transmission } \\
\text { from an infectious Human to } \\
\text { Mosquito }\end{array}$ & 0.24 & Chitnis $N$ et al. (2008) [37] \\
\hline$\beta_{H}$ & Infection Rate of Humans & 0.00638 & Osman et al. (2017) [1] \\
\hline$\beta_{V}$ & Infection Rate of Mosquitoes & 0.0696 & Osman et al. (2017) [1] \\
\hline$\delta$ & $\begin{array}{l}\text { Disease Induced Death Rate in } \\
\text { Humans }\end{array}$ & 0.0681 & Assumed \\
\hline$\psi$ & $\begin{array}{l}\text { Newborn's birth rate with } \\
\text { infection }\end{array}$ & 0.0003 & Osman et al. (2017) [1] \\
\hline$\rho$ & Loss of immunity in Humans & 0.00017 & $\begin{array}{l}\text { Ishikawa H. [36] } \\
\text { et al. (2013) }\end{array}$ \\
\hline
\end{tabular}

\subsubsection{VIM Application to Model Equations}

We have the correction functional of the system of equation governing this malaria model as: 


$$
\begin{aligned}
& S_{(n+1) H}(t)=S_{n H}(t)+\int_{0}^{t} \pi_{1}(x)\left[S_{n H}^{\prime}+\beta_{H} \tilde{S}_{n H} \tilde{I}_{n H}+\mu_{H} S_{n H}-\Lambda_{H}-\rho R_{n H}\right] d x \\
& E_{(n+1) H}(t)=E_{n H}(t)+\int_{0}^{t} \pi_{2}(x)\left[E_{n H}^{\prime}+\left(\alpha_{1}+\mu_{H}\right) E_{n H}-\beta_{H} \tilde{S}_{n H} \tilde{I}_{n H}\right] d x \\
& I_{(n+1) H}(t)=I_{n H}(t)+\int_{0}^{t} \pi_{3}(x)\left[I_{n H}^{\prime}+\left(\alpha_{2}+\mu_{H}+\delta-\psi\right) I_{n H}-\alpha_{1} E_{n H}\right] d x \\
& R_{(n+1) H}(t)=R_{n H}(t)+\int_{0}^{t} \pi_{4}(x)\left[R_{n H}^{\prime}+\left(\mu_{H}+\rho\right) R_{n H}-\alpha_{2} I_{n H}\right] d x \\
& S_{(n+1) V}(t)=S_{n V}(t)+\int_{0}^{t} \pi_{5}(x)\left[S_{n V}^{\prime}+\mu_{V} S_{n V}+\beta_{n V} \tilde{S}_{n V} \tilde{I}_{n V}-\Lambda_{V}\right] d x \\
& E_{(n+1) V}(t)=E_{n V}(t)+\int_{0}^{t} \pi_{6}(x)\left[E_{n V}^{\prime}+\left(\alpha_{3}+\mu_{V}\right) E_{n V}-\beta_{n V} \tilde{S}_{n V} \tilde{I}_{n V}\right] d x \\
& I_{(n+1) V}(t)=I_{n V}(t)+\int_{0}^{t} \pi_{7}(x)\left[I_{n V}^{\prime}+\mu_{V} I_{n V}-\alpha_{3} E_{n V}\right] d x
\end{aligned}
$$

Subject to the initial conditions

$$
\begin{gathered}
S_{H}(0)=0.83, E_{H}(0)=0.08, I_{H}(0)=0.07, R_{H}(0)=0.02 S_{V}(0)=0.7, E_{V}(0)=0.2, I_{V}(0)=0.1 \\
\pi_{1}(x)=\pi_{2}(x)=\pi_{3}(x)=\pi_{4}(x)=\cdots=\pi_{7}(x)=-1
\end{gathered}
$$

By putting the model parameters and the value of the lagrangian multiplier in eq. (72); we obtain an iteration formula for the seven (7) compartments as:

$$
\begin{aligned}
& S_{(n+1) H}(t)=S_{n H}(t)-\int_{0}^{t}\left[S_{n H}^{\prime}+0.00638 S_{n H} I_{n H}+0.0115 S_{n H}-1.2-0.00017 R_{n H}\right] d x \\
& E_{(n+1) H}(t)=E_{n H}(t)-\int_{0}^{t}\left[E_{n H}^{\prime}+0.0615 E_{n H}-0.00638 S_{n H} I_{n H}\right] d x \\
& I_{(n+1) H}(t)=I_{n H}(t)-\int_{0}^{t}\left[I_{n H}^{\prime}+0.0801 I_{n H}-0.05 E_{n H}\right] d x \\
& R_{(n+1) H}(t)=R_{n H}(t)-\int_{0}^{t}\left[R_{n H}^{\prime}+0.01167 R_{n H}-0.0035 I_{n H}\right] d x \\
& S_{(n+1) V}(t)=S_{n V}(t)-\int_{0}^{t}\left[S_{n V}^{\prime}+0.05 S_{n V}+0.696 S_{n V} I_{n V}-0.7\right] d x \\
& E_{(n+1) V}(t)=E_{n V}(t)-\int_{0}^{t}\left[E_{n V}^{\prime}+0.133 E_{n V}-0.0696 S_{n V} I_{n V}\right] d x \\
& I_{(n+1) V}(t)=I_{n V}(t)-\int_{0}^{t}\left[I_{n V}^{\prime}+0.05 I_{n V}-0.083 E_{n V}\right] d x
\end{aligned}
$$

We obtain the iterated values for each population/compartments as: 
$\mathrm{S}_{\mathrm{H}}(\mathrm{t})=0.83+1.190087722 \mathrm{t}-0.007104328345 \mathrm{t}^{2}+0.00003194682064 t^{3}$ $-7.001923802 \times 10^{-7} t^{4}+8.456685994 \times 10^{-10} t^{5}+1.163198684 \times 10^{-12} t^{6}$ $-3.423963937 \times 10^{-15} t^{7}+5.342315808 \times 10^{-16} t^{8}-3.723617710 \times 10^{-18} t^{9}$ $+6.322821679 \times 10^{-21} t^{10}-2.271139500 \times 10^{-24} t^{11}+\cdots$

$E_{H}(t)=0.08-0.004549322000 \mathrm{t}+0.0004012165808 \mathrm{t}^{2}-0.00001258921184 \mathrm{t}^{3}$ $+0.1061638566 \times 10^{-5} \mathrm{t}^{4}-6.061655945 \times 10^{-9} \mathrm{t}^{5}+1.270625328 \times 10^{-11} \mathrm{t}^{6}$ $-2.077566160 \times 10^{-15} \mathrm{t}^{7}-5.342315808 \times 10^{-16} \mathrm{t}^{8}+3.723617710 \times 10^{-18} \mathrm{t}^{9}$ $-6.322821679 \times 10^{-21} \mathrm{t}^{10}+2.271139500 \times 10^{-24} t^{11}+\cdots$

$I_{H}(t)=0.07-0.001607000000 t-0.00004937270000 t^{3}-1.370365576 \times 10^{-7} t^{4}$ $+1.788543167 \times 10^{-8} t^{5}-1.196734896 \times 10^{-10} t^{6}+2.494651094 \times 10^{-13} t^{7}$ $-6.806885860 \times 10^{-17} t^{8}-2.967953227 \times 10^{-18} t^{9}+1.861808855 \times 10^{-20} t^{10}+\cdots$

$R_{H}(t)=0.02+0.0000116000000 \mathrm{t}-0.2879936000 \times 10^{-5} \mathrm{t}^{2}-4.30259846 \times 10^{-8} \mathrm{t}^{3}$ $-2.017061262 \times 10^{-9} \mathrm{t}^{4}-3.199583573 \times 10^{-10} \mathrm{t}^{5}+2.406919418 \times 10^{-18} \mathrm{t}^{8}$ $-6.934725980 \times 10^{-15} \mathrm{t}^{7}+3.028880838 \times 10^{-12} \mathrm{t}^{6}$

$S_{V}(\mathrm{t})=0.7+0.6601280000 \mathrm{t}-0.01908302144 \mathrm{t}^{2}+2.040227226 \times 10^{-4} t^{3}$ $+1.303653606 \times 10^{-5} t^{4}+1.519901404 \times 10^{-6} t^{5}+{ }_{-} 2.043333541 \times 10^{-7} t^{6}$ $+6.022746410 \times 10^{-9} t^{7}-7.190253944 \times 10^{-10} t^{8}+1.796296797 \times 10^{-11} t^{9}$ $-2.018406198 \times 10^{-13} t^{10}-2.474577295 \times 10^{-14} t^{11}+9.727190967 \times 10^{-16} t^{12}+\cdots$ $E_{V}(t)=0.2-0.02172800000 t+0.004024733440 t^{2}+0.0000435937856 t^{3}$ $-7.18167845 \times 10^{-6} t^{4}+3.93765013 \times 10^{-7} t^{5}-2.744923850 \times 10^{-7} t^{6}$ $-1.432467516 \times 10^{-9} t^{7}+6.147220304 \times 10^{-10} t^{8}-2.319266431 \times 10^{-11} t^{9}$ $+2.479625495 \times 10^{-13} t^{10}+2.651694009 \times 10^{-14} t^{11}+\cdots$ 


$$
I_{V}(t)=0.1+0.01160000 t-0.001191712000 t^{2}+0.0001312128252 t^{3}
$$

$-2.97650627 \times 10^{-6} t^{4}+3.88118335 \times 10^{-7} t^{5}+6.846185837 \times 10^{-8} t^{6}$

$-2.099291186 \times 10^{-9} t^{7}-7.998304105 \times 10^{-12} t^{8}+2.968697857 \times 10^{-13} t^{9}$

$+2.512090497 \times 10^{-15} t^{10}-7.265313407 \times 10^{-17} t^{11}+\cdots$

This gives the Semi-analytic solution of the SEIR-SEI model of malaria Transmission.

\subsection{Numerical Results}

The analytical Results for the SEIR-SEI model is illustrated and demonstrated in this section.

This results were achieved using Computer Software and by using the parameter values and state variable values in the table 2 whose source were mainly from prominent literatures as well as assumptions.

The results obtained by our proposed VIM were compared with the Computer In-built Rungekutta felhberg of fourth-fifth order (RKF-45) with degree four interpolant. In which a table of values obtained from all compartments is presented.

Table 3 and 4 presents the results comparison of $S_{H}(t), E_{H}(t), I_{H}(t), R_{H}(t), S_{V}(t), E_{V}(t)$, and $I_{V}(t)$ between VIM and RKF-45.

5.1 Table 3: The Host (Human) Population Model

Comparison between Variational iteration Method and Runge-Kutta-Felhberg-45 (VIM vs RKF45)

\begin{tabular}{lllcccc}
\hline TIME & VIM & RKF-45 & VIM & RKF-45 & VIM & RKF-45 \\
\cline { 2 - 7 } Day $(\mathrm{s})$ & $S_{H}(t)$ & $S_{H}(t)$ & $E_{H}(t)$ & $E_{H}(t)$ & $I_{H}(t)$ & $I_{H}(t)$ \\
0 & $\mathbf{0 . 8 3 0 0 0 0 0 0}$ & $\mathbf{0 . 8 3 0 0 0 0 0 0}$ & $\mathbf{0 . 0 8 0 0 0 0 0 0}$ & $\mathbf{0 . 0 8 0 0 0 0 0 0}$ & $\mathbf{0 . 0 7 0 0 0 0 0 0}$ & $\mathbf{0 . 0 7 0 0 0 0 0 0}$ \\
1 & $\mathbf{2 . 0 1 3 0 1 5 6 1}$ & $\mathbf{2 . 0 1 3 0 1 5 6 1}$ & $\mathbf{0 . 0 7 5 8 3 8 7 8}$ & $\mathbf{0 . 0 7 5 8 3 8 7 9}$ & $\mathbf{0 . 0 6 8 3 5 1 3 1}$ & $\mathbf{0 . 0 6 8 3 5 1 3 3}$ \\
2 & $\mathbf{3 . 1 8 2 0 1 6 2 1}$ & $\mathbf{3 . 1 8 2 0 1 6 1 7}$ & $\mathbf{0 . 0 7 2 4 0 1 9 9}$ & $\mathbf{0 . 0 7 2 4 0 1 9 8}$ & $\mathbf{0 . 0 6 6 6 4 7 5 3}$ & $\mathbf{0 . 0 6 6 6 4 7 5 4}$ \\
3 & $\mathbf{4 . 3 3 7 1 9 3 6 1}$ & $\mathbf{4 . 3 3 7 1 9 3 5 4}$ & $\mathbf{0 . 0 6 9 6 1 5 5 1}$ & $\mathbf{0 . 0 6 9 6 1 5 5 0}$ & $\mathbf{0 . 0 6 4 9 2 5 8 8}$ & $\mathbf{0 . 0 6 4 9 2 5 9 0}$ \\
4 & $\mathbf{5 . 4 7 8 7 3 6 8 3}$ & $\mathbf{5 . 4 7 8 7 3 6 7 7}$ & $\mathbf{0 . 0 6 7 4 1 0 3 8}$ & $\mathbf{0 . 0 6 7 4 1 0 3 7}$ & $\mathbf{0 . 0 6 3 2 1 7 2 9}$ & $\mathbf{0 . 0 6 3 2 1 7 3 0}$ \\
5 & $\mathbf{6 . 6 0 6 8 3 1 0 4}$ & $\mathbf{6 . 6 0 6 8 3 1 0 4}$ & $\mathbf{0 . 0 6 5 7 2 3 3 2}$ & $\mathbf{0 . 0 6 5 7 2 3 3 2}$ & $\mathbf{0 . 0 6 1 5 4 7 1 2}$ & $\mathbf{0 . 0 6 1 5 4 7 1 3}$ \\
6 & $\mathbf{7 . 7 2 1 6 5 7 4 3}$ & $\mathbf{7 . 7 2 1 6 5 7 4 3}$ & $\mathbf{0 . 0 6 4 4 9 6 8 1}$ & $\mathbf{0 . 0 6 4 4 9 6 8 4}$ & $\mathbf{0 . 0 5 9 9 3 5 9 0}$ & $\mathbf{0 . 0 5 9 9 3 5 8 9}$ \\
7 & $\mathbf{8 . 8 2 3 3 9 2 3 7}$ & $\mathbf{8 . 8 2 3 3 9 2 3 7}$ & $\mathbf{0 . 0 6 3 6 7 9 2 6}$ & $\mathbf{0 . 0 6 3 6 7 9 3 4}$ & $\mathbf{0 . 0 5 8 3 9 9 9 9}$ & $\mathbf{0 . 0 5 8 3 9 9 8 9}$ \\
8 & $\mathbf{9 . 9 1 2 2 0 5 8 2}$ & $\mathbf{9 . 9 1 2 2 0 7 5 9}$ & $\mathbf{0 . 0 6 3 2 2 4 6 3}$ & $\mathbf{0 . 0 6 3 2 2 4 9 6}$ & $\mathbf{0 . 0 5 6 9 5 2 2 4}$ & $\mathbf{0 . 0 5 6 9 5 1 8 6}$ \\
9 & $\mathbf{1 0 . 9 8 8 2 6 9 9 4}$ & $\mathbf{1 0 . 9 8 8 2 6 9 9 3}$ & $\mathbf{0 . 0 6 3 0 9 2 3 1}$ & $\mathbf{0 . 0 6 3 0 9 3 3 7}$ & $\mathbf{0 . 0 5 5 6 0 2 6 0}$ & $\mathbf{0 . 0 5 5 6 0 1 4 7}$ \\
10 & $\mathbf{1 2 . 0 5 1 7 4 1 4 4}$ & $\mathbf{1 2 . 0 5 1 7 4 1 4 5}$ & $\mathbf{0 . 0 6 3 2 4 6 5 6}$ & $\mathbf{0 . 0 6 3 2 4 9 5 3}$ & $\mathbf{0 . 0 5 4 3 5 8 8 0}$ & $\mathbf{0 . 0 5 4 3 5 5 8 4}$ \\
\hline
\end{tabular}




\begin{tabular}{lrl}
\hline TIME & \multicolumn{1}{l}{ VIM } & \multicolumn{1}{l}{ RKF-45 } \\
\cline { 2 - 3 } Day(s) & $R_{H}(t)$ & $R_{H}(t)$ \\
0 & $\mathbf{0 . 0 2 0 0 0 0 0 0}$ & $\mathbf{0 . 0 2 0 0 0 0 0 0}$ \\
1 & $\mathbf{0 . 0 2 0 0 0 8 6 8}$ & $\mathbf{0 . 0 2 0 0 0 8 6 8}$ \\
2 & $\mathbf{0 . 0 2 0 0 1 1 4 2}$ & $\mathbf{0 . 0 2 0 0 1 1 4 2}$ \\
3 & $\mathbf{0 . 0 2 0 0 0 8 1 5}$ & $\mathbf{0 . 0 2 0 0 0 8 1 5}$ \\
4 & $\mathbf{0 . 0 1 9 9 9 8 9 5}$ & $\mathbf{0 . 0 1 9 9 9 8 9 5}$ \\
5 & $\mathbf{0 . 0 1 9 9 8 3 9 6}$ & $\mathbf{0 . 0 1 9 9 8 3 9 6}$ \\
6 & $\mathbf{0 . 0 1 9 9 6 3 4 4}$ & $\mathbf{0 . 0 1 9 9 6 3 4 4}$ \\
7 & $\mathbf{0 . 0 1 9 9 3 7 6 8}$ & $\mathbf{0 . 0 1 9 9 3 7 6 8}$ \\
8 & $\mathbf{0 . 0 1 9 9 0 7 0 2}$ & $\mathbf{0 . 0 1 9 9 0 7 0 2}$ \\
9 & $\mathbf{0 . 0 1 9 8 7 1 8 4}$ & $\mathbf{0 . 0 1 9 8 7 1 8 4}$ \\
10 & $\mathbf{0 . 0 1 9 8 3 2 5 6}$ & $\mathbf{0 . 0 1 9 8 3 2 5 6}$ \\
\hline
\end{tabular}

5.2 Table 4: The Vector (Mosquito) Population Model Comparison between Variational iteration Method and Runge-Kutta-Felhberg-45 (VIM vs RKF45)

\begin{tabular}{|c|c|c|c|c|c|c|}
\hline TIME & VIM & RKF-45 & VIM & RKF-45 & VIM & RKF-45 \\
\hline & $S_{V}(t)$ & $S_{V}(t)$ & $E_{V}(t)$ & $E_{V}(t)$ & $I_{V}(t)$ & $I_{V}(t)$ \\
\hline 0 & 0.70000000 & 0.70000000 & 0.20000000 & 0.20000000 & 0.10000000 & 0.10000000 \\
\hline 1 & 1.34126043 & 1.34126044 & 0.18222063 & 0.18222068 & 0.11053636 & 0.11053636 \\
\hline 2 & 1.94571554 & 1.94571556 & 0.17196734 & 0.17196734 & 0.11943083 & 0.11943067 \\
\hline 3 & 2.51484441 & 2.51484462 & 0.16860461 & 0.16860489 & 0.12734893 & 0.12734679 \\
\hline 4 & 3.05014134 & 3.05014133 & 0.17149462 & 0.17149495 & 0.13486396 & 0.13486393 \\
\hline 5 & 3.55302285 & 3.55302267 & 0.18008280 & 0.18008282 & 0.14248463 & 0.14248433 \\
\hline 6 & 4.02476836 & 4.02476839 & 0.19394581 & 0.19394865 & 0.15064626 & 0.15064609 \\
\hline 7 & 4.46648246 & 4.46648299 & 0.21276299 & 0.21283345 & 0.16002117 & 0.15973839 \\
\hline 8 & 4.87907270 & 4.87907287 & 0.23664713 & 0.23664911 & 0.17011741 & 0.17011742 \\
\hline 9 & 5.26322652 & 5.26323337 & 0.26472804 & 0.26547877 & 0.18212468 & 0.18212189 \\
\hline 10 & 5.61944102 & 5.61944155 & 0.28884690 & 0.29957234 & 0.19608738 & 0.19608754 \\
\hline
\end{tabular}


Multiple plot profiles of all compartments in the model (Graphical illustrations)

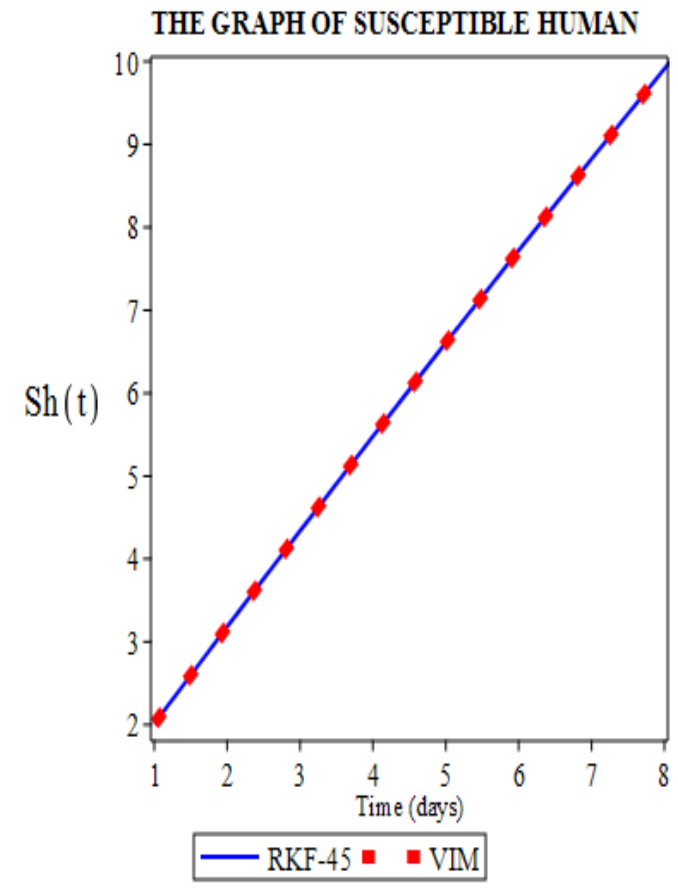

Fig 1 Solution comparison plot of the susceptible human population between variational iteration method and Runge-Kutta-Felhberg 45 (RKF-45).

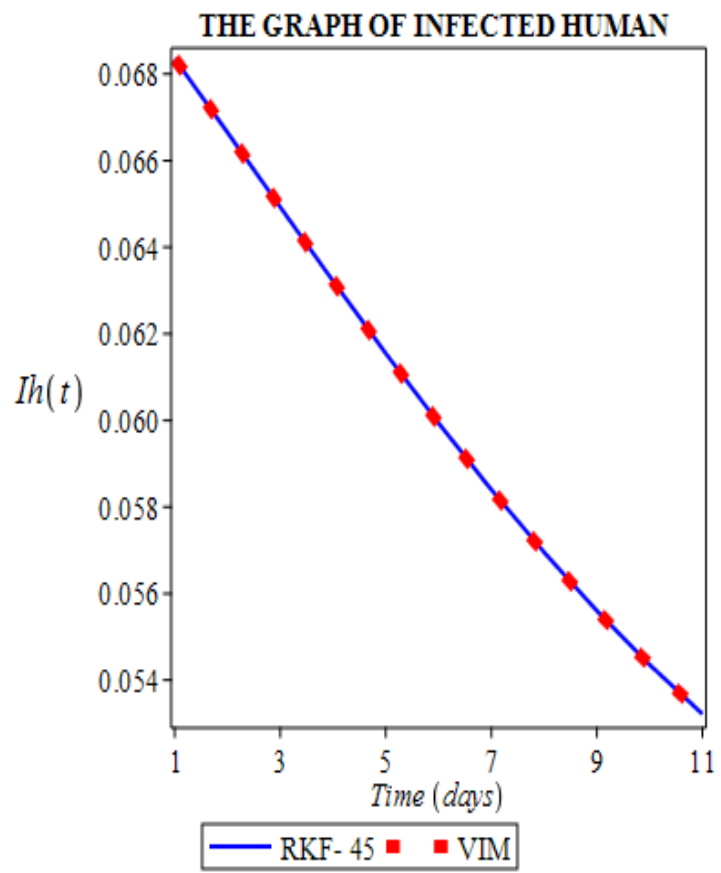

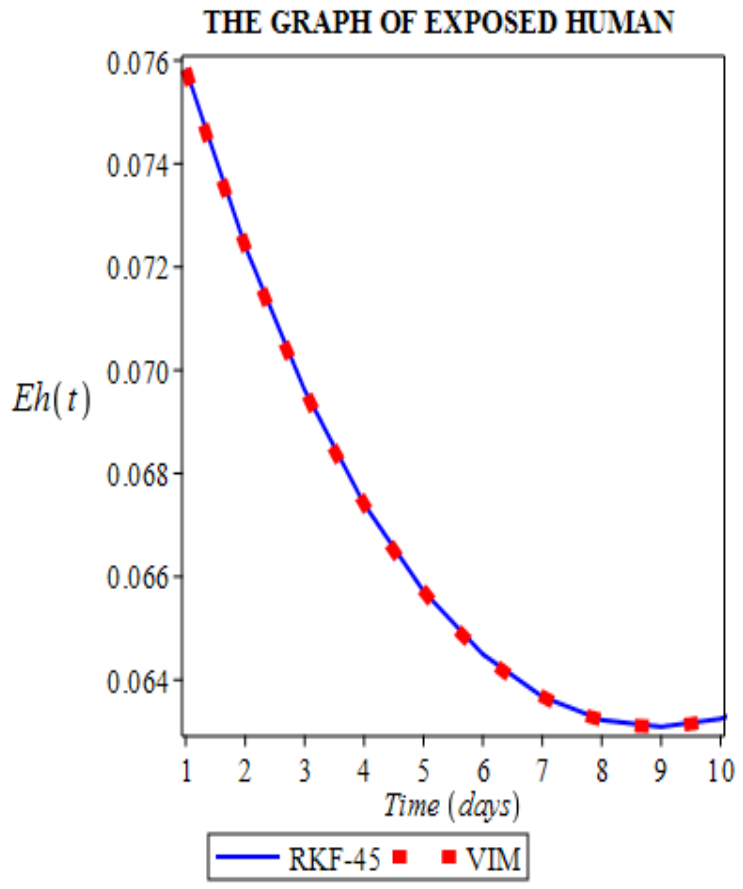

Fig 2 Solution comparison plot of the exposed population between variational Iteration method (VIM) and Runge-KuttaFelhberg 45 (RKF-45).

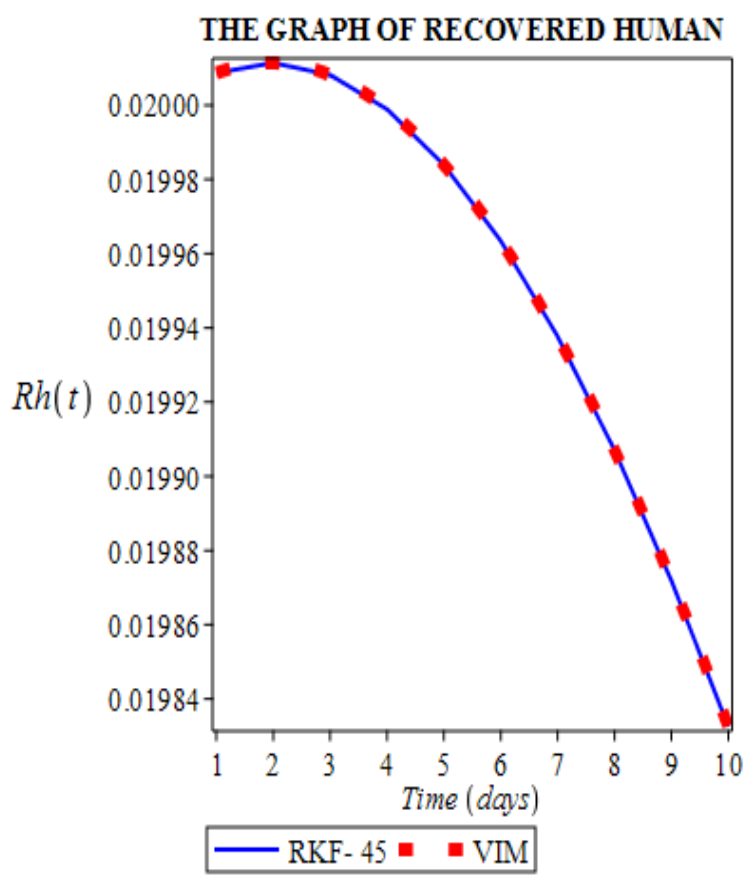


Fig 3 Solution comparison plot of the Infected human population between Variational iteration method (VIM) and Runge-Kutta-felhberg 45 (RKF-45)

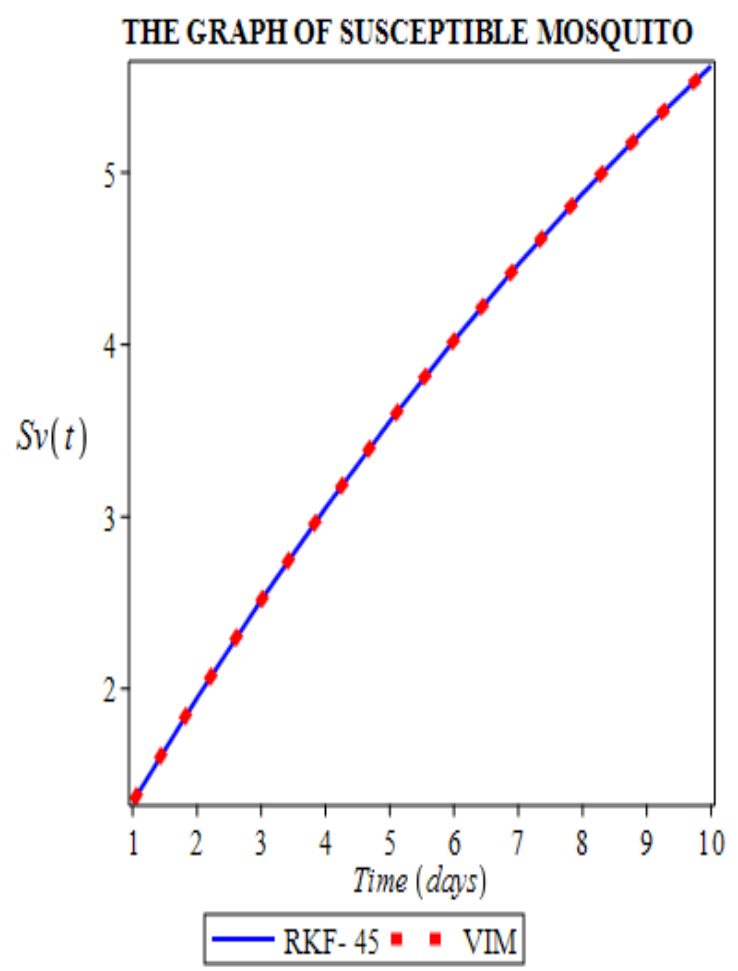

Fig 5 Solution comparison plot of the Susceptible mosquito between variational Iteration method (VIM) and Runge-KuttaFelhberg 45 (RKF-45)
Fig 4 Solution comparison plot of the recovered human population between variational iteration method and RungeKutta-felhberg 45 (RKF-45)

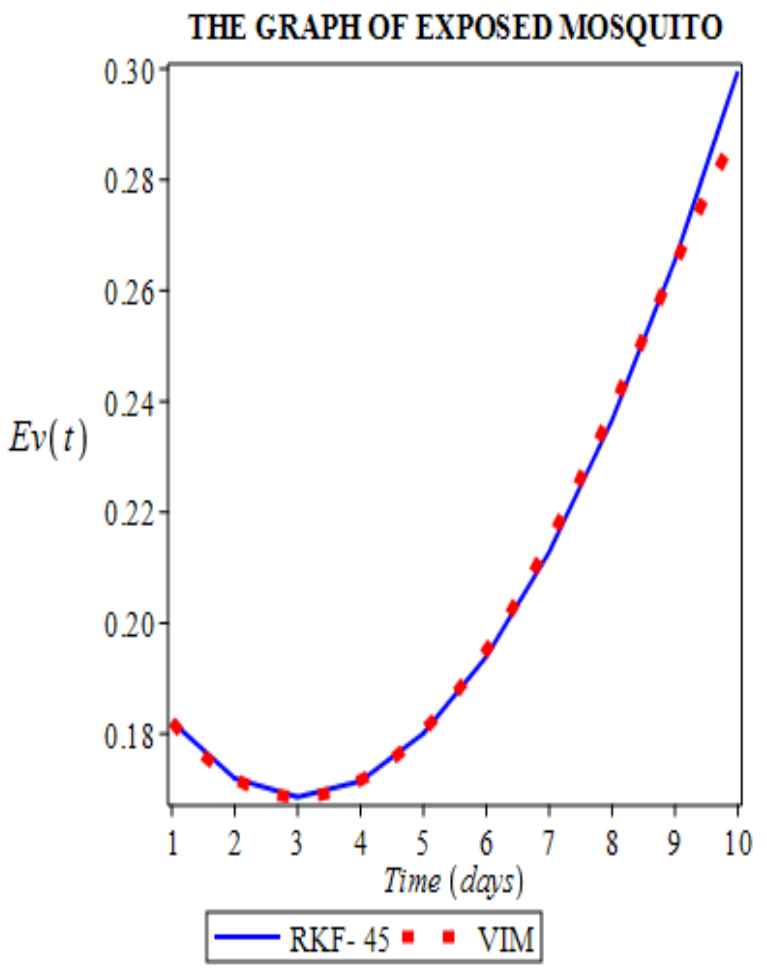

Fig 6 Solution comparsion plot of the exposed mosquitoes between variational iteration method and Runge-Kutta-felhberg 


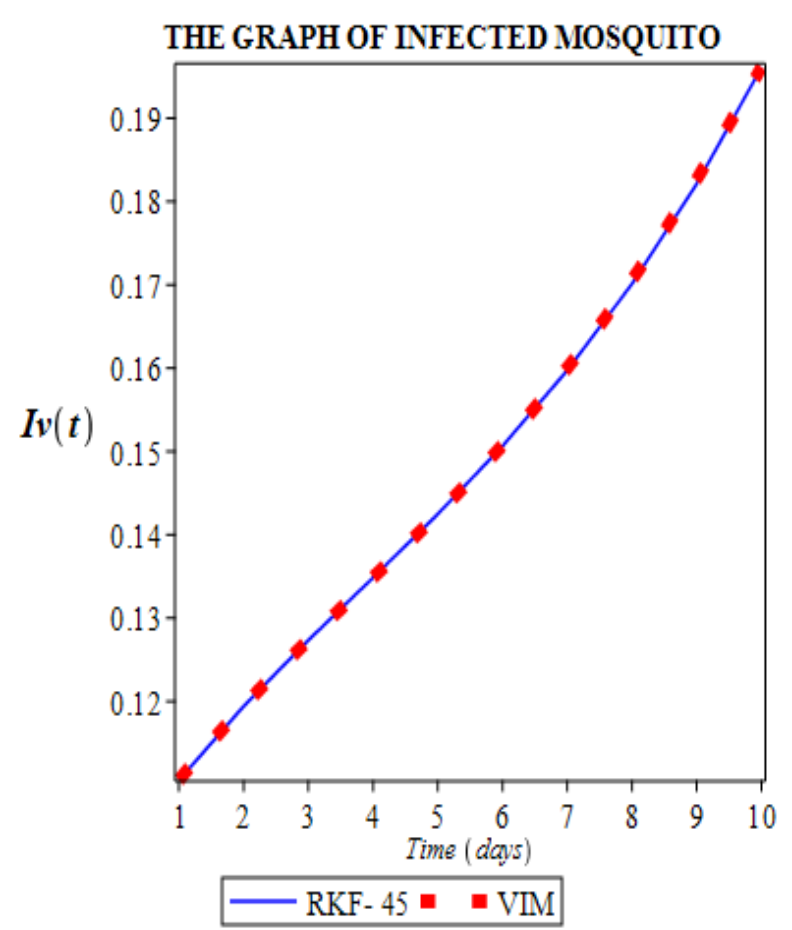

Fig 7 Solution comparison plot of the infected

Mosquito between variational iteration method (VIM)

And Runge-Kutta-Felhberg 45 (RKF-45)

\subsection{Interpretation of Results}

The Results obtained from the Numerical simulation and the stability analysis of the proposed SEIR-SEI Vector-host Malaria Transmission model shows that the Disease-free equilibrium is stable when a mosquito doesn't infect more than one individual. That is, when $R_{0}$ does not exceed unity $\left(R_{0}<1\right)$; and moment the converse happens (when $R_{0}>1$ ) then there is an epidemic outbreak (endemic equilibrium).

Furthermore, the population of the susceptible human undergoes an exponential growth pattern, and the graph is perfectly linear.

The Population of the exposed and the infected human decays (decreases) in a logistic manner and hence a logistic decay which implies that there is a possibility of zero population (At DFE). While the Recovered Human, Susceptible, Exposed and infected mosquito follows a logistic growth pattern.

All these imply that the populations in the system are prone to the infection since the population in the susceptible compartment is increasing. 
As a result, serious attention should be focused on this compartment as regards an appropriate intervention strategy to combat the contact of malaria infection at a time when the infected and the exposed population is stable (when $R_{0}<1$ ).

\subsection{Discussion of Results}

A SEIR-SEI Vector-Host model of malaria transmission built on 7-dimensional system of ordinary differential was analyzed and solved numerically using the Variational iteration method (VIM) with initial conditions and parameter values from prominent existing literature.

The stability analyses show that there is a possibility of the malaria infection going into extinction if one mosquito does not infect more than one individual (when $R_{0}<1$ ). Similarly, epidemic outbreak of the disease is visible and might occur when $R_{0}>1$.

The Semi-analytical solution to the Malaria model using VIM when compared favorably with the Computer software in-built Runge-Kutta-Felhberg of fourth-fifths order (RKF-45) shows a high level of agreement, convergence and similarities. The two methods follow the same pattern and behavior when plotted.

Having used our proposed method, it is now crystal clear that the variational iteration method is suitable, perfect and efficient in conducting and conveying analysis on Malaria models.

\subsection{Conclusion}

We have studied a nonlinear 7-dimensional ordinary differential equation that describes the transmission dynamics of malaria by carrying asymptotic stability analyses at the malaria-free and endemic equilibriums using the Gaussian elimination method and the routh-hurwitz criterion with three (3) theorems and three (3) lemmas. We also have solved the model using the variational iteration method (VIM). This method is unprecedented.

From all these, we have come to a conclusion that the variational is an efficient alternative for conducting analyses on malaria models and other epidemiological models and as a matter of fact can be implemented in the classroom to provide solutions to a wider class of ordinary differential equations and epidemiological models.

\subsection{Declaration of Interest}

Authors have declared that there is no conflict of interest relevant to this research 


\section{References}

[1] Mojeeb AL-Rahman EL-Nor Osman, Isaac Kwasi Adu, and Cuihong Yang, A Simple SEIR Mathematical Model of Malaria transmission, Asian Research journal of Mathematics 7(3) (2017), pp. 1-22.

[2] Olaniyi S and Olaniyi OS, Mathematical model for malaria transmission dynamics on human and mosquito population with nonlinear forces of infectious disease, Int. Journal of Pure and Applied Mathematics, 88(1) (2013), pp. 125-150.

[3] Jia Li, Modelling of transgenic mosquitoes and impact on malaria transmission, Journal of Biological Dynamics, 5:5 (2011), pp. 474-494, DOI: $\underline{10.1080 / 17513758.2010 .523122}$

[4] Ross R. Report on the prevention of Malaria in Mauritius. London (1908). Available from https://archive.org/details/onpreventi00rossreportrich [Google scholar]

[5] Macdonald G (1957), The Epidemiology and control of Malaria. Oxford University Press, London

[6] Driessche PV and Watmough J, Reproduction numbers and sub-threshold endemic equilibria for Compartmental models of disease transmission, Mathematical Biosciences 180 (2002) pp. 29-48.

[7] Traoré Bakary, Sangaré Boureima \& Traoré Sado, A mathematical model of malaria transmission in a periodic environment, Journal of Biological Dynamics, 12:1 (2018), pp. 400432, DOI: $\underline{10.1080 / 17513758.2018 .1468935}$

[8] Alejandra Ortiz-Ruiz, Maria postigo, Sara Gil-casanova, Daniel Cuadrado, Jose M. Bautista, Jose Miguel Rubio, Miguel Luengo-Oroz and Maria Linares, Plasmodium Species Differentiation by Non-expert on-line volunteers for Remote Malaria field Diagnosis, BMC Malaria Journal 17(54) (2018).

[9] WHO World Malaria Report (2013), Available at https://www.who.int/malaria/publications/world_malaria_report_2013/en/ [10] WHO World Malaria Report (2015), Available at https://www.who.int/malaria/publications/world-malaria-report-2015/report/en/ 
[11] WHO World Malaria Report (2010) Available at https://www.who.int/malaria/world malaria report 2010/en/

[12] WHO World Malaria Report (2016) Available at https://www.who.int/malaria/publications/world-malaria-report-2016/report/en/

[13] Getachew Geleta and Tsige Ketema, Severe Malaria Associated with Plasmodium falciparum and P. vivax among Children in Pawe Hospital, Northwest Ethiopia, Malaria Research and Treatment, vol. $2016 \quad$ (2016.), Article ID 1240962, 7 pages, https://doi.org/10.1155/2016/1240962.

[14] Ruhani Yusof, Yee Ling Lau, Rohela Mahmud, Mun Yik Fong, Jenarun Jelip,... and Marlindawati Mohd Ali, High Proportion of Knowlesi Malaria in recent Malaria cases in Malaysia, BMC Malaria Journal 13(168) (2014).

[15] Caraballo, H. and King, K., Emergency Department Management of Mosquito-Borne Illness: Malaria, Dengue, and West Nile Virus. Emergency Medicine Practice, 16 (2014), pp. 123.

[16] Fact sheet on the World Malaria Report (2014) Available at https://www.who.int/malaria/media/world_malaria_report_2014/en/

[17] Haq Fazal, Shah Kamal, Khan Asaf, Shahzad Muhammad and Rahman Ghaus, Numerical Solution of Fractional Order Epidemic Model of a Vector Born Disease by Laplace Adomian Decomposition Method. Punjab University Journal of Mathematics. 49(10) (2017).

[18] Ngwa G.A, Shu W.S. A mathematical model for endemic with variable human and mosquito Populations, Available:www.ictp.trieste.it/pub.off.1999

[19] Rangkuti, Yulita Molliq; Side, Syafruddin; Noorani, Mohd Salmi Md. Numerical Analytic Solution of SIR Model of Dengue Fever Disease in South Sulawesi using Homotopy Perturbation Method and Variational Iteration Method. Journal of Mathematical and Fundamental Sciences, 46(1) (2014), pp. 91-105. Available at: <http://journals.itb.ac.id/index.php/jmfs/article/view/819 $>$. doi:10.5614/j.math.fund.sci.2014.46.1.8. 
[20] Goswami P, Murty U.S, Mutheneni S.R, Kukkuthady A, Krishnan S.T, A Model of Malaria Epidemiology involving Weather, Exposure and Transmission applied to North East India. PloS One, 7(11) (2012).

[21] Shah NH, Gupta J. SEIR model and simulation for vector borne diseases, Applied Mathematics. 4(2013), pp. 13-17.

[22] Altaf Khan M, Abdul Wahid, Saeed Islam, IIyas Khan, Sharidan Shafie, and Taza Gul, Stability analysis of an SEIR Model with non-linear saturated incidence and temporary immunity. Int. J. Adv. Appl. Math and Mech., 2(3) (2015):234-7-2529.

[23] Hai-Feng Huo and Guang-Ming Qiu, Stability of a Mathematical Model of Malaria Transmission with Relapse, Abstract and Applied Analysis, vol. 2014 (2014), Article ID 289349, 9 pages. https://doi.org/10.1155/2014/289349.

[24] Mojeeb AL-Rahman EL-Nor Osman, Appiagyei Ebenezer and Isaac Kwasi Adu, A Simple SEIR Mathematical Model of Malaria transmission, Journal of Advances in Mathematics and Computer science 28(6) (2018), pp. 1-17.

[25] Nigar Ali \& Saeed Ahmad \& Sartaj Aziz \& Gul Zaman,, The Adomian Decomposition Method For Solving HIV Infection Model Of Latently Infected Cells, Matrix Science Mathematic (MSMK), Zibeline International Publishing, vol. 3(1) (2019), pp. 5-8.

[26] O, Olayiwola \& Kayode Kolawole, Mutairu \& Popoola, Amos, Variational Iteration Method for the Simulation of the Effect of Transmission Coefficient on the SusceptibleExposed-Infected-Recovered-Susceptible (Seirs) Epidemic Model with Saturated Incidence Rate and Disease-Induced Death. 17 (2017). Pp. 357-364.

[27] Abioye, Adesoye \& Ibrahim, Mohammed Olanrewaju \& Olumuyiwa, Peter \& Sylvanus, Amadiegwu \& Festus, Oguntolu. Differential Transform Method for Solving Mathematical Model of SEIR and SEI Spread of Malaria. 40 (2018). Pp. 197-219. 
[28] He, J. H, Variational iteration method a kind of nonlinear analytical technique: Some examples, Int. J. Nonlinear Mech., Vol.34 (1999), Pp 669-708.

[29] Olumuyiwa, P., Folasade, A.A., Abiodun, O.F., Sambo, B., \& Emmanuel, A.C. Multistep Homotopy Analysis Method for Solving Malaria Model, Malaysian Journal of Applied Sciences, 3(2) (2018).

[30] G. Birkhoff and G. C. Rota, Ordinary Differential Equations, Ginn, Boston, 1982.

[31] Inokuti, M., General Use of the Lagrange Multiplier in Nonlinear Mathematical Physics. In: Nemat Nasser, S., Ed., Variational Method in the Mechanics of Solid, Pergamon Press, Oxford, (1978) pp. 156-162.

[32] Abbasbandy, E \& Shivanian, E., Application of the variational iteration method for systemof nonlinear Volterra'sintegro-differential equations, Mathematical and Comp. Applic., Vol 14, Issue2 (2009), Pp 147-158.

[33] Momani, S,Abuasad, S (2006), Application of He's variational iteration method toHelmholtz equation, Chaos, Solitons\& Fractals., 27: 1119- 1123strategies in microbial pathogenesis, Nature, 430, 250-256.

[34] Abdou, M . A \& Soliman, A. A, Variational iteration method for solving Burger's and coupled Burger's equation, J. Comp. Appl. Math., Vol 181, Issue 2 (2005), 245-251.

[35] Jia Li. New revised simple models for interactive Wild and sterile Mosquito Populations and their Dynamics. Journal of Biological Dynamics. 2015, 34:D25i92D;92D40.

[36] Chitnis N, Hyman JM, Cushing JM. Determining important parameters in the spread of malaria through the sensitivity analysis of a mathematical model. Bulletin of Mathematical Biology 200870 pp. 1272-1296 
[37] Ishikawa $\mathrm{H}$, et al. A mathematical model for the transmission of Plasmodium vivax malaria, Parasitology International 52 (2013) pp. 81-93.

[38] WHO World Malaria Report (2018) Available at
https://www.who.int/malaria/publications/world-malaria-report-2018/en/

[39] 2019 Commonwealth Malaria Report Available at https://www.malariamustdie.com/sites/default/files/201904/CommonwealthMalariaReport2019.pdf

[40] Ji-Huan He, Homotopy perturbation method for solving boundary value problems, Physics Letters A, 350(2): 87-88, (2006) https://doi.org/10.1016/j.physleta.2005.10.005.

Available at: (http://www.sciencedirect.com/science/article/pii/S0375960105015434)

[41] Deniz, Sinan \& Bildik, Necdet, A Note on Stability Analysis of Taylor Collocation Method, Celal Bayar Üniversitesi Fen Bilimleri Dergisi, 10.18466/cbayarfbe.302660, (2017)

[42] Bildik, Necdet \& Deniz, Sinan, The Use of Sumudu Decomposition Method for Solving Predator-Prey Systems. Mathematical Sciences Letters. 5. 285-289. 10.18576/ms1/050310, (2016)

[43] Alvaro Salas H., solving nonlinear partial differential equations by the sn-ns method, Abstract and Applied Analysis, Article ID 340824: 25 pages, (2012)

DOI: $\underline{\text { https://doi.org/10.1155/2012/340824. }}$

[44] Loyinmi, A.C., Akinfe, T.K. An algorithm for solving the Burgers-Huxley equation using Elzaki transform, SN Applied sciences, 2(7), (2020)

DOI: https://doi.org/10.1007/s42452-019-1653-3

[45] Nadeem Muhammad, Li Fengquan and Ahmad Hijaz, Modified Laplace variational iteration method for solving fourth-order parabolic partial differential equation with variable coefficients. Computers and Mathematics with Applications. 10.1016/j.camwa.2019.03.053, (2019)

[46] He, Ji-Huan., Homotopy perturbation method: A new nonlinear analytical technique. Applied Mathematics and Computation - AMC. 135. 73-79. 10.1016/S0096-3003(01)00312-5, (2003) 
[47] He, Ji-Huan., Application of homotopy perturbation method to nonlinear wave Equations. Chaos, Solitons \& Fractals. 26. 695-700. 10.1016/j.chaos.2005.03.006, (2005)

[48] Aziz Imra, Islam Siraj-ul-Islam, and Asif Muhammad, Haar wavelet collocation method for three-dimensional elliptic partial differential equations. Computers and Mathematics with Applications. 73. 10.1016/j.camwa.2017.02.034, (2017)

[49] Loyinmi, A.C. \& Akinfe, T.K.:Exact solution to the family of Fisher's reaction diffusion equation using Elzaki Homotopy transformation perturbation method. Engineering Reports. 2019; e12084. DOI: https://doi.org/10.1002/eng2.12084 